\title{
Analysis of the saturated electromagnetic devices under DC bias condition by the decomposed harmonic balance finite element method
}

\begin{abstract}
Purpose - This paper aims to introduce the decomposed harmonic balance finite element method (HBFEM) to decrease the memory requirement in large-scale computation of the DC-biasing magnetic field. Harmonic analysis of the flux density and flux distribution was carried out to investigate the DC biased problem in a laminated core model (LCM).

Design/methodology/approach - Based on the DC bias test on LCM, the decomposed HBFEM is applied to calculate the DC-biasing magnetic field effectively. External electric circuits are coupled with the magnetic field in the form of harmonic. The reluctivity matrix is decomposed and each harmonic solution of magnetic field and exciting current is solved by the block Gauss-Seidel algorithm sequentially.

Findings - The calculated exciting currents and flux density is compared with that obtained from measurement and time domain finite element analysis respectively, which demonstrates a good consistency. The DC bias leads to the significant saturation of the magnetic core and serious distortion of the exciting current. The flux density varies nonlinearly with DC bias excitation.

Research limitations/implications - The harmonic balance method is applicable to solve the steady state magnetic field only, and the future improvement of the method is to handle the hysteresis effects in magnetic material.

Originality/value - The proposed method to solve the DC biased problem reduces memory requirement greatly compared with the traditional HBFEM. The decomposed harmonic balance equations are solved efficiently by the block Gauss-Seidel algorithm and over-relaxation iterative scheme. Investigation on DC bias phenomena is carried out through the harmonic solution of magnetic field. The decomposed HBFEM can be applied to solve the DC-biasing magnetic field in a practical power transformer based on the methodology presented in this paper.
\end{abstract}

Keywords Harmonic balance, LCM, DC bias, Flux density

\section{Introduction}

Electromagnetic devices such as electrical machines and power transformers are generally operated under steady-state conditions. Owing to the nonlinearity of magnetic material in electromagnetic devices, there often are high-order harmonics in the exciting current and magnetic field. The HBFEM has been proposed to solve the steady-state magnetic field with eddy current problems (Yamada et al., 1988; Yamada et al. 1989; Bachinger et al., 2006) and design the switching power supplies (Lu et al., 1996). A modified method via block decomposition of the system equation has been presented to reduce the memory requirement (Yamada et al., 1991), though DC component and even harmonics are not considered in computation. The HBFEM has been further developed by introducing differential reluctivity tensor (Gyselinck et al., 2002) and transmission-line modeling technique (Deblecker et al., 2006) respectively, and the magnetic field of a three phase transformer was calculated.

The DC biased problem of power transformers has been paid more attention in recent years (Picher et al., 1997; Yao et al., 2005; Biro et al., 2008). The magnetic storm and HVDC transmission system may generate large quasi-direct and direct current respectively on the earth (Pirjola et al., 2000; Zhang et al., 2006). The direct currents flow into the windings through the 
grounded neutral points of transformers, which leads to large harmonics in exciting currents, significant saturation of the ferromagnetic core, mechanical vibration and overheating of the transformer (Tay et al., 1985;), reactive power demand in the transmission system (You et al., 1996) and even crash of the electric network (Bolduc et al., 2002). Harmonic analysis of exciting current and flux density has been done by the traditional HBFEM to investigate the DC bias phenomena (Zhao et al., 2010). However, the drawback of large memory requirement in HBFEM has limited its widespread application in the large-scale computation.

This paper introduces the decomposed HBFEM to calculate the DC-biasing magnetic field of LCM in harmonic domain, considering the harmonic coupling between the external circuits and magnetic field. The magnetic reluctivity matrix can be separated in the form of harmonic, thus the system matrix are decomposed and solved sequentially for each harmonic. A modified resolution scheme for the nonlinear equation set is developed at the same time. More detailed analysis of the flux density and flux distribution under different DC bias conditions are carried out through the harmonic solutions of magnetic field.

\section{Decomposed harmonic balanced system equation}

The following vector potential equation can be used to describe the two dimensional magnetic field, where $A$ and $J$ are magnetic vector potential and current density respectively, and $v$ is the magnetic reluctivity,

$$
\nabla \times(\nu \nabla \times A)=J .
$$

Due to the periodic characteristics of the electromagnetic field under DC bias condition, the steady state variables such as current density $J$, magnetic vector potential $A$, and flux density $B$ can be approximated by a triangular series, in which the DC component, odd and even harmonics coexist.

$$
\begin{gathered}
J^{m}(t)=J_{0}^{m}+\sum_{i=1}^{\infty}\left(J_{2 i-1}^{m} \sin i \omega t+J_{2 i}^{m} \cos i \omega t\right), \\
A^{m}(t)=A_{0}^{m}+\sum_{i=1}^{\infty}\left(A_{2 i-1}^{m} \sin i \omega t+A_{2 i}^{m} \cos i \omega t\right), \\
B_{x}^{e}(t)=B_{x, 0}^{e}+\sum_{i=1}^{\infty}\left(B_{x, 2 i-1}^{e} \sin i \omega t+B_{x, 2 i}^{e} \cos i \omega t\right), \\
B_{y}^{e}(t)=B_{y, 0}^{e}+\sum_{i=1}^{\infty}\left(B_{y, 2 i-1}^{e} \sin i \omega t+B_{y, 2 i}^{e} \cos i \omega t\right),
\end{gathered}
$$

where the superscript $m$ means the node number in one triangle element $e$, the symbol $i$ and $\omega$ represent the harmonic number and fundamental angular frequency.

The magnetic reluctivity $v$, which is a function of magnetic flux density $B$, can also be expressed by

$$
v(t)=H(t) / B(t)=f(B(t))=v_{0}+\sum_{i=1}^{\infty}\left(v_{2 i-1} \sin i \omega t+v_{2 i} \cos i \omega t\right) .
$$

The decomposed finite element equation can be obtained by substituting equations (2)-(6) into equation (1), 


$$
\begin{gathered}
\left\{\begin{array}{l}
\left(\boldsymbol{S}^{e} * \boldsymbol{R}_{1,1}^{e}+\boldsymbol{T}^{e} * \boldsymbol{h}_{1}\right) \boldsymbol{A}_{1}^{e}=-\sum_{j=1,2,3 \ldots}^{j \neq 1}\left(\boldsymbol{S}^{e} * \boldsymbol{R}_{1, j}^{e}\right) \boldsymbol{A}_{j}^{e}+\boldsymbol{K}_{1}^{e} \\
\vdots \\
\left(\boldsymbol{S}^{e} * \boldsymbol{R}_{i, i}^{e}+\boldsymbol{T}^{e} * \boldsymbol{h}_{i}\right) \boldsymbol{A}_{i}^{e}=-\sum_{j=1,2,3 \ldots}^{j \neq 1}\left(\boldsymbol{S}^{e} * \boldsymbol{R}_{i, j}^{e}\right) \boldsymbol{A}_{j}^{e}+\boldsymbol{K}_{i}^{e} \\
\vdots
\end{array}\right. \\
\boldsymbol{A}_{i}^{e}=\left\{\begin{array}{l}
\left\{A_{0}^{1}, A_{1}^{1}, A_{2}^{1}, A_{0}^{2}, A_{1}^{2}, A_{2}^{2}, A_{0}^{3}, A_{1}^{3}, A_{2}^{3}\right\} \quad(i=1) \\
\left\{A_{i}^{1}, A_{i+1}^{1}, A_{i}^{2}, A_{i+1}^{2}, A_{i}^{3}, A_{i+1}^{3}\right\} \quad(i=2, \ldots N)
\end{array},\right. \\
\boldsymbol{K}_{i}^{e}=\left\{\begin{array}{l}
(\Delta / 3)\left\{J_{0}^{1}, J_{1}^{1}, J_{2}^{1}, J_{0}^{2}, J_{1}^{2}, J_{2}^{2}, J_{0}^{3}, J_{1}^{3}, J_{2}^{3}\right\}^{T} \quad(i=1) \\
(\Delta / 3)\left\{J_{i}^{1}, J_{i+1}^{1}, J_{i}^{2}, J_{i+1}^{2}, J_{i}^{3}, J_{i+1}^{3}\right\} \quad(i=2, \ldots N)
\end{array},\right.
\end{gathered}
$$

where $\mathfrak{R}_{i, i}^{e}$ and $\boldsymbol{h}_{i}$ represent the self-correlation reluctivity matrix and the harmonic matrix respectively related to the $i$-th harmonic solution of magnetic vector potential $\boldsymbol{A}_{i}^{e} \cdot \mathfrak{R}_{i, j}^{e}$ represents the cross-correlation reluctivity related to the $i$-th and $j$-th harmonic. $\boldsymbol{S}^{e}$ is the coefficient matrix related to the magnetic reluctivity, and $\boldsymbol{T}^{e}$ is the coefficient matrix relevant to the eddy current. $\boldsymbol{K}_{i}$ is obtained from the spatial distribution of the $i$-th harmonic component of current density. $\Delta$ is the area of one triangular element and $N$ is the truncated harmonic number.

The DC component of magnetic vector potential is coupled with the fundamental harmonic in computation, therefore the expression of self-correlation reluctivity matrix $\mathfrak{R}_{i, i}^{e}(i=1)$ differs from the other ones $\Re_{i, i}^{e}(i>1)$ shown in equation (10). It is the same with the cross-correlation reluctivity matrix $\mathfrak{R}_{i, j}^{e}$, which is expressed by equations (11), (12) and (13),

$$
\begin{aligned}
& \mathfrak{R}_{i, i}^{e}=\left\{\begin{array}{l}
{\left[\begin{array}{ccc}
2 v_{0} & v_{1} & v_{2} \\
2 v_{1} & 2 v_{0}-v_{4} & v_{3} \\
2 v_{2} & v_{3} & 2 v_{0}+v_{4}
\end{array}\right](i=1),} \\
{\left[\begin{array}{cc}
2 v_{0}-v_{4 i} & v_{4 i-1} \\
v_{4 i-1} & 2 v_{0}+v_{4 i}
\end{array}\right] \quad(i>1)}
\end{array}\right. \\
& \boldsymbol{R}_{1, i}^{e}=\frac{1}{2}\left[\begin{array}{cc}
v_{2 i-1} & v_{2 i} \\
v_{2 i-2}-v_{2 i+2} & v_{2 i+1}-v_{2 i-3} \\
v_{2 i+1}+v_{2 i-3} & v_{2 i-2}+v_{2 i+2}
\end{array}\right](i>1), \\
& \left(\mathscr{R}_{i, 1}^{e}\right)^{T}=\frac{1}{2}\left[\begin{array}{cc}
2 v_{2 i-1} & 2 v_{2 i} \\
v_{2 i-2}-v_{2 i+2} & v_{2 i+1}-v_{2 i-3} \\
v_{2 i+1}+v_{2 i-3} & v_{2 i-2}+v_{2 i+2}
\end{array}\right](i>1), \\
& \mathfrak{R}_{i, j}^{e}=\left(\boldsymbol{R}_{j, i}^{e}\right)^{T}=\left[\begin{array}{cc}
v_{2\left(m^{\prime}-n^{\prime}\right)}-v_{2\left(m^{\prime}+n^{\prime}\right)} & v_{2\left(m^{\prime}-n^{\prime}\right)-1}+v_{2\left(m^{\prime}-n^{\prime}\right)+1} \\
v_{2\left(m^{\prime}-n^{\prime}\right)+1}-v_{2\left(m^{\prime}-n^{\prime}\right)-1} & v_{2\left(m^{\prime}-n^{\prime}\right)}+v_{2\left(m^{\prime}+n^{\prime}\right)}
\end{array}\right](i, j>1 \& i \neq j),
\end{aligned}
$$

where the subscripts $m^{\prime}$ and $n^{\prime}$ are relevant to the harmonic number $i$ and $j(i, j>1$ and $i \neq j) . m^{\prime}$ is defined by $\operatorname{Max}(i, j)$ and $n^{\prime}$ by $\operatorname{Min}(i, j)$.

The harmonic matrix $\boldsymbol{h}_{i}$ is also different because of the addition of DC component and even harmonics in computation, 


$$
\boldsymbol{h}_{i}=\left\{\begin{array}{l}
{\left[\begin{array}{ccc}
0 & 0 & 0 \\
0 & 0 & -1 \\
0 & 1 & 0
\end{array}\right](i=1)} \\
\omega\left[\begin{array}{cc}
0 & -i \\
i & 0
\end{array}\right] \quad(i>1)
\end{array} .\right.
$$

The operator $*$ in equation (7) can be defined by

$$
\boldsymbol{S}^{e} * \boldsymbol{R}_{i, i}^{e}+\boldsymbol{T}^{e} * \boldsymbol{h}_{i}=\left[\begin{array}{ccc}
S_{11} \boldsymbol{R}_{i, i}^{e} & S_{12} \boldsymbol{R}_{i, i}^{e} & S_{13} \boldsymbol{R}_{i, i}^{e} \\
S_{21} \boldsymbol{R}_{i, i}^{e} & S_{22} \boldsymbol{R}_{i, i}^{e} & S_{23} \boldsymbol{R}_{i, i}^{e} \\
S_{31} \boldsymbol{R}_{i, i}^{e} & S_{32} \boldsymbol{R}_{i, i}^{e} & S_{33} \boldsymbol{R}_{i, i}^{e}
\end{array}\right]+\left[\begin{array}{lll}
T_{11} \boldsymbol{h}_{i} & T_{12} \boldsymbol{h}_{i} & T_{13} \boldsymbol{h}_{i} \\
T_{21} \boldsymbol{h}_{i} & T_{22} \boldsymbol{h}_{i} & T_{23} \boldsymbol{h}_{i} \\
T_{31} \boldsymbol{h}_{i} & T_{32} \boldsymbol{h}_{i} & T_{33} \boldsymbol{h}_{i}
\end{array}\right],
$$

where $S_{m, n}$ is the element of the coefficient matrix $S^{e}$ in the finite element region $\Omega_{e}$, while $T_{m, n}$ means the element of the coefficient matrix $\boldsymbol{T}^{e} . S_{m, n}$ and $T_{m, n}$ can be defined by the following expressions,

$$
\begin{gathered}
S_{m, n}=\int_{\Omega_{e}} \nabla N_{m} \cdot \nabla N_{n} d \Omega, \\
T_{m, n}=\int_{\Omega_{e}} \sigma N_{m} \cdot N_{n} d \Omega,
\end{gathered}
$$

where $N_{m}$ and $N_{n}$ are the interpolation functions. The subscripts $m$ and $n$ represent the node number in the triangular element $(m, n=1,2,3)$, and $\sigma$ is the conductivity.

By assembling all triangular elements in the computational region, the system matrix in the form of separated harmonic can be written as for simplicity,

$$
\boldsymbol{Q}_{i} \boldsymbol{A}_{i}=\boldsymbol{K}_{i}+\boldsymbol{F}_{i} \quad(i=1,2, \ldots, N)
$$

where

$$
\left\{\begin{array}{l}
\boldsymbol{F}_{i}=-\sum_{j=1,2,3 \ldots}^{j \neq 1}\left(\boldsymbol{S} * \boldsymbol{R}_{i, j}\right) \boldsymbol{A}_{j} \\
\boldsymbol{Q}_{i}=\boldsymbol{S} * \boldsymbol{R}_{i, i}+\boldsymbol{T} * \boldsymbol{h}_{i}
\end{array}\right.
$$

\section{Magnetic field coupled with electric circuits}

When electromagnetic devices are excited by voltage sources, the electric potential difference in the coil region can be obtained from the following equation (Lombard et al., 1992),

$$
V_{k}=R_{k} I_{k}+L_{k} \frac{d I_{k}}{d t}+\frac{1}{C_{k}} \int I_{k} d t+\frac{N_{k} l_{k}}{S_{k}} \int_{\Omega_{c}}(\partial A / \partial t) d \Omega,
$$

where $V_{k}$ is the input voltage of circuit $k$, and $I_{k}$ is the corresponding exciting current. $R_{k}$ and $S_{k}$ are the resistance and cross-sectional area of the $k$-th winding respectively. $L_{k}$ is the inductance and $C_{k}$ is capacitance of external circuit $k . N_{k}$ is the turn number of the $k$-th winding, and $l_{k}$ is the thin wire's length in z-direction. In the DC-biasing case the input voltage can be written as follows,

$$
V_{k}=V_{k, 0}+\sum_{i=1}^{\infty}\left(V_{k, 2 i-1} \sin i \omega t+V_{k, 2 i} \cos i \omega t\right) .
$$

Hence the harmonics in voltages, currents and magnetic vector potentials can be expressed separately by the following equation,

$$
\boldsymbol{V}_{k, i}=\boldsymbol{Z}_{k, i} \boldsymbol{J}_{k, i} S_{k}+\left(N_{k} l_{k} / S_{k}\right) \int_{\Omega_{c}}\left(\partial \boldsymbol{A}_{i} / \partial t\right) d \Omega .
$$




$$
\boldsymbol{V}_{k, i}=\left\{\begin{array}{l}
\left\{V_{k, 0}, V_{k, 1}, V_{k, 2}\right\}^{T} \quad(i=1) \\
\left\{V_{k, 2 i-1}, V_{k, 2 i}\right\}^{T} \quad(i>1)
\end{array}\right.
$$

The subscripts $i$ in equation (22) indicates the harmonic number. $\boldsymbol{V}_{k, i}$ and $\boldsymbol{J}_{k, i}$ are the $i$-th harmonic vectors of the input voltage and the current density in circuit $k$, respectively. Nonlinear components in external circuits can be expressed in the form of harmonic impedance matrices as follows,

$$
\boldsymbol{Z}_{k, i}=\left[\begin{array}{cc}
R_{k}+i \omega L_{k}-\left(i \omega C_{k}\right)^{-1} & 0 \\
0 & R_{k}-i \omega L_{k}+\left(i \omega C_{k}\right)^{-1}
\end{array}\right]
$$

Combined with finite element method, equation (20) can be represented by equations (25) and (26),

$$
\begin{gathered}
\boldsymbol{V}_{k, i}=\boldsymbol{Z}_{k, i} \boldsymbol{J}_{k, i} S_{k}+\boldsymbol{C}_{k, i} \boldsymbol{A}_{i}, \\
\boldsymbol{C}_{k, i}=\sum_{n=1}^{N_{c}} \frac{\omega N_{k} l_{k}}{3 S_{k}}\left[\begin{array}{lll}
\boldsymbol{h}_{i} & \boldsymbol{h}_{i} & \boldsymbol{h}_{i}
\end{array}\right] .
\end{gathered}
$$

where the matrix $\boldsymbol{C}_{k, i}$ is the coupled matrix linking the electric circuit $k$ with the magnetic field. $N_{c}$ is the finite element number in coil region. The harmonic solutions of magnetizing currents and magnetic vector potentials are obtained by solving equations (18) and (25) together, which is shown by the following equation,

$$
\left[\begin{array}{cc}
\boldsymbol{Q}_{i} & \boldsymbol{G}_{k, i} \\
\boldsymbol{C}_{k, i} & \boldsymbol{Z}_{k, i}
\end{array}\right]\left\{\begin{array}{c}
\boldsymbol{A}_{i} \\
\boldsymbol{J}_{k, i}
\end{array}\right\}=\left\{\begin{array}{c}
\boldsymbol{F}_{i} \\
\boldsymbol{V}_{k, i}
\end{array}\right\}(i=1,2, \ldots, N),
$$

where the matrix $\boldsymbol{G}_{k, i}$ is related to the spatial distribution of the $i$-th harmonic component of the current density. Finally $N$ separated equations shown in (27) constitute the system equation when $N$ harmonics are considered in the computation.

\section{Computational procedure based on the block Gauss-Seidel algorithm}

\subsection{Previous calculation procedure}

In Yamada et al. (1991), a calculation procedure to solve the system equation (18) has been proposed:

1) The fundamental harmonic $\boldsymbol{A}_{1}$ is calculated at first based on the assumption of setting $\boldsymbol{A}_{2} \sim$ $\boldsymbol{A}_{N}$ to be zero. If the convergence of $\boldsymbol{A}_{1}$ is satisfied, turn to 2). If not, update $\boldsymbol{A}_{1}$ and repeat 1).

2) The other harmonics $\boldsymbol{A}_{2} \sim \boldsymbol{A}_{N}$ are calculated respectively with the known $\boldsymbol{A}_{1}$. If the convergence of $\boldsymbol{A}_{2} \sim \boldsymbol{A}_{N}$ is satisfied, stop. If not, turn to 1).

However, the computational procedure above is not dependable and efficient. The convergent criterion in each harmonic computation and no full use of updated solutions in nonlinear iterations lead to the uncertainty of convergence and the subsequent inaccuracy of harmonic solutions especially when the strongly nonlinear magnetic material is involved. Yamada et al also pointed out that there is uncertainty of the convergence in the iterative approach. Actually a compulsory stop is usually done by setting a maximum iterative number.

\subsection{Improved computational procedure}

The nonlinear terms $\boldsymbol{Q}_{i}$ and $\boldsymbol{F}_{i}$ in equation (27) are obtained from the self-correlation reluctivity 
matrix $\mathfrak{R}_{i, i}$ and cross-correlation reluctivity matrix $\boldsymbol{R}_{i, j}$ respectively. It can be seen from equations (10)-(13) that diagonal elements in matrix $\mathfrak{R}_{i, i}$ are mainly expressed by $v_{0}$ (the DC component of reluctivity), while the elements in matrix $\boldsymbol{R}_{i, j}$ are defined by $v_{\mathrm{n}}$ (the harmonic components of magnetic reluctivity). The harmonic components of magnetic reluctivity $v_{\mathrm{n}}$ are much smaller than the DC component $v_{0}$ in several orders of magnitude. Therefore diagonally dominant characteristic exists in the system equation (27) and the block Gauss-Seidel algorithm (Saad et al., 2009) can be used to solve the DC-biasing magnetic field. The new computational procedure is as follows:

1) $(p=0)$ : Initialize $\boldsymbol{A}_{1}^{0}, \boldsymbol{A}_{2}^{0} \ldots \boldsymbol{A}_{\mathrm{N}}^{0}$ and solve the decomposed harmonic balance equation set in (27) following the procedure at step 2).

2) $(p>0)$ :

a). (i=1): Update $\boldsymbol{Q}_{1}^{\mathrm{p}}$ by $\boldsymbol{A}_{1}^{\mathrm{p}}, \boldsymbol{A}_{2}^{\mathrm{p}}, \ldots, \boldsymbol{A}_{\mathrm{N}}^{\mathrm{p}}$ and $\boldsymbol{F}_{1}^{\mathrm{p}}$ by $\boldsymbol{A}_{2}^{\mathrm{p}}, \boldsymbol{A}_{3}^{\mathrm{p}}, \ldots, \boldsymbol{A}_{\mathrm{N}}^{\mathrm{p}}$. Assemble the matrices $\boldsymbol{C}_{1}$ and $\boldsymbol{G}_{1}$. Solve the equations (27) to obtain the renewed fundamental harmonic vector $\boldsymbol{A}_{1}^{\mathrm{p}+1}$.

b). $(1<i<N)$ : Update $\boldsymbol{Q}_{\mathrm{i}}^{\mathrm{p}}$ by $\boldsymbol{A}_{1}^{\mathrm{p}}, \ldots, \boldsymbol{A}_{\mathrm{i}}^{\mathrm{p}}, \ldots, \boldsymbol{A}_{\mathrm{N}}^{\mathrm{p}}$ and $\boldsymbol{F}_{\mathrm{i}}^{\mathrm{p}}$ by $\boldsymbol{A}_{1}^{\mathrm{p}+1}, \boldsymbol{A}_{2}^{\mathrm{p}+1}, \ldots, \boldsymbol{A}_{\mathrm{i}-1}^{\mathrm{p}+1}, \boldsymbol{A}_{\mathrm{i}+1}^{\mathrm{p}}, \ldots, \boldsymbol{A}_{\mathrm{N}}^{\mathrm{p}}$. Assemble the matrices $\boldsymbol{C}_{\mathrm{i}}$ and $\boldsymbol{G}_{\mathrm{i}}$. Solve the $i$-th equation in (27) to obtain the renewed $i$-th harmonic vector $\boldsymbol{A}_{\mathrm{i}}^{\mathrm{p}+1}$.

c). $(i=N)$ : Update $\boldsymbol{Q}_{\mathrm{N}}^{\mathrm{p}}$ by $\boldsymbol{A}_{1}^{\mathrm{p}}, \ldots, \boldsymbol{A}_{\mathrm{N}-1}^{\mathrm{p}}, \boldsymbol{A}_{\mathrm{N}}^{\mathrm{p}}$ and $\boldsymbol{F}_{\mathrm{N}}^{\mathrm{p}}$ by $\boldsymbol{A}_{1}^{\mathrm{p}+1}, \boldsymbol{A}_{2}^{\mathrm{p}+1}, \ldots, \boldsymbol{A}_{\mathrm{N}-1}^{\mathrm{p}+1}$. Assemble the matrices $\boldsymbol{C}_{\mathrm{N}}$ and $\boldsymbol{G}_{\mathrm{N}}$. Solve the $N$-th equation in (27) to obtain the renewed $N$-th harmonic vector $\boldsymbol{A}_{\mathrm{N}}^{\mathrm{p}+1}$.

3) Check for convergence criterion. If satisfied, stop. Otherwise, renew all harmonic solutions with a properly selected over-relaxation factor, $A^{\text {new }}=(1-\alpha) A^{\text {now }}+\alpha A^{\text {old }}$, and return to step 2) for the next iteration $(p=p+1)$.

where the symbol $i$ means the harmonic number in the computation. $N$ is the truncated harmonic number and $p$ represents iterative number. The symbol $\alpha$ is a properly selected over-relaxation factor aiming at rapid convergence of the harmonic solution in nonlinear iterations. The convergence condition is defined by

$$
\left|\frac{A_{i}^{p}(m)-A_{i}^{p-1}(m)}{A_{\max }}\right|<\varepsilon_{1} \text { and }\left|\frac{J_{i}^{p}(m)-J_{i}^{p-1}(m)}{J_{\max }}\right|<\varepsilon_{2} \quad(i=1,2, \ldots, N)
$$

where $A_{\max }$ and $J_{\max }$ represents the maximum value of all harmonic solutions $\boldsymbol{A}$ and current density $J$ respectively.

\subsection{Comparison between previous method and the new method}

Compared with the previous calculation procedure in 4.1 , the improved computational approach in 4.2 makes the harmonic solutions converge smoothly and unconditionally. The Gauss-Seidel algorithm can be used to solve the nonlinear magnetic field equations more efficiently.

When the DC-biasing magnetic field is calculated, suppose that there are $M$ nodes in the finite element region, thus comparison of memory required in computation can be indicated by the largest matrix size as follows,

$$
\frac{M S_{N}}{M S_{T}}=\frac{[3 \times M]^{2}}{[(2 N+1) \times M]^{2}}=\frac{3 \times 3}{(2 N+1)^{2}},
$$

where $M S_{N}$ represents the matrix size of $\boldsymbol{Q}_{1}$ in equation (27) while $M S_{T}$ represents the system matrix size in traditional HBFEM (Yamada et al., 1990; Zhao et al., 2010). Therefore the memory 
requirement has been reduced greatly by the decomposed harmonic balance equations, which is suitable for large-scale computation.

\section{DC-biasing test on the LCM and computational results}

The square LCM shown in Figure 1 was made by Tianwei Group, Baoding, China. The magnetic core is entwined by two coils of 312 turns. One is the exciting coil, and the other is the searching coil. The model was tested under different DC bias conditions. The DC bias $I_{d c}$ from a DC current source is applied to the exciting coils, and the no-load induced voltage $U_{a c}$ of $50 \mathrm{~Hz}$ is measured by the searching coil. In the test the DC bias is smaller than 3 amperes since too large DC bias may damage the LCM. Figure 2 shows the schematic circuit diagram of the DC-biasing test.

The exciting currents and DC-biasing magnetic field are computed simultaneously by the block Gauss-Seidel algorithm proposed in 4.2. The over-relaxation factor $\alpha$ is initially set to be 0.2 (Cheng et al. 2009) and then varies with the iterative number $P_{c}$. An alternative scheme is shown in Table I in detail. The waveforms of calculated exciting currents shown in Figure 3 are satisfactory in comparison with the measured results. That proves the effectiveness of the decomposed HBFEM to solve the DC biased problem. The computational results under different DC bias excitations are listed partly in Table II. $P_{c}$ and $N$ represents the iterative number for convergence and truncated harmonic number respectively. $I_{r m s, c}$ is the root-mean-square value of calculated exciting current, while $I_{r m s, m}$ is the counterpart obtained from measurement.

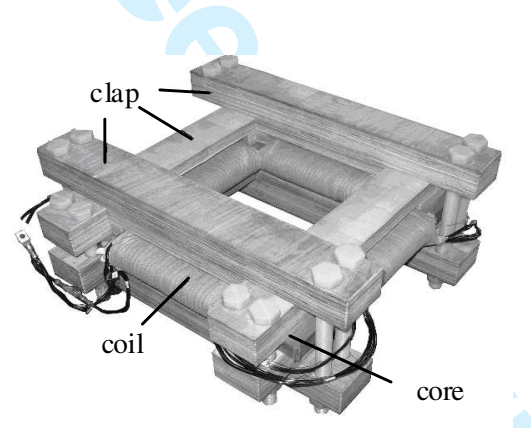

Figure 1. Laminated core model

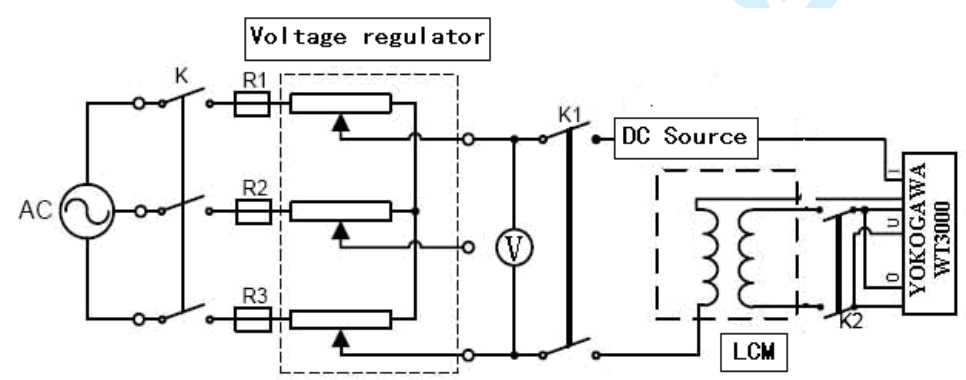

Figure 2. Schematic circuit diagram of the core model in experiment

Table I Scheme of setting the over-relaxation factor for convergence of harmonic solution

\begin{tabular}{|c|c|c|c|c|c|c|c|c|c|}
\hline$P_{\mathrm{c}}$ & $<10$ & $<20$ & $<40$ & $<60$ & $<70$ & $<80$ & $<90$ & $<100$ & $\geq 100$ \\
\hline$\beta$ & 1 & 0.8 & 0.6 & 0.4 & 0.3 & 0.2 & 0.1 & 0.08 & 0.06 \\
\hline$\alpha=\alpha \cdot \beta$ & 0.2 & 0.16 & 0.12 & 0.08 & 0.06 & 0.04 & 0.02 & 0.016 & 0.012 \\
\hline
\end{tabular}


Table II Calculation results under different DC bias excitations $\left(\varepsilon_{1}=\varepsilon_{2}=0.005\right)$

\begin{tabular}{ccccccc}
\hline \hline$I_{\mathrm{dc}}(\mathrm{A})$ & $U_{\mathrm{ac}}(\mathrm{V})$ & $N$ & $P_{\mathrm{c}}$ & $I_{\mathrm{rms}, \mathrm{c}}(\mathrm{A})$ & $I_{\mathrm{rms}, \mathrm{m}}(\mathrm{A})$ & Error $(\%)$ \\
0.4256 & 370 & 9 & 396 & 1.2943 & 1.3486 & 4.03 \\
0.4256 & 240 & 9 & 190 & 1.0861 & 1.1264 & 3.58 \\
0.4256 & 185 & 9 & 161 & 1.0219 & 0.9855 & 3.56 \\
0.847 & 240 & 9 & 360 & 2.3337 & 2.3463 & -0.53 \\
\hline \hline
\end{tabular}

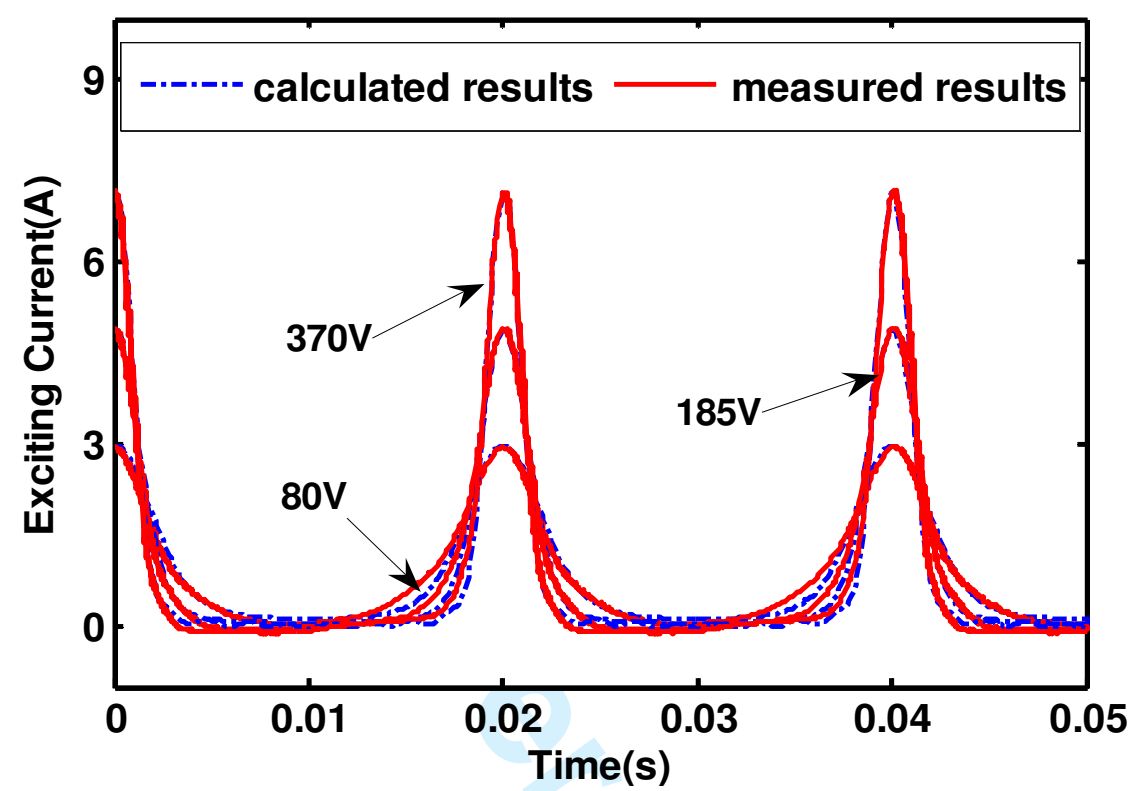

(a) $I_{d c}=0.84 \mathrm{~A} ; U_{a c}=80 \mathrm{~V}, 185 \mathrm{~V}, 370 \mathrm{~V}$

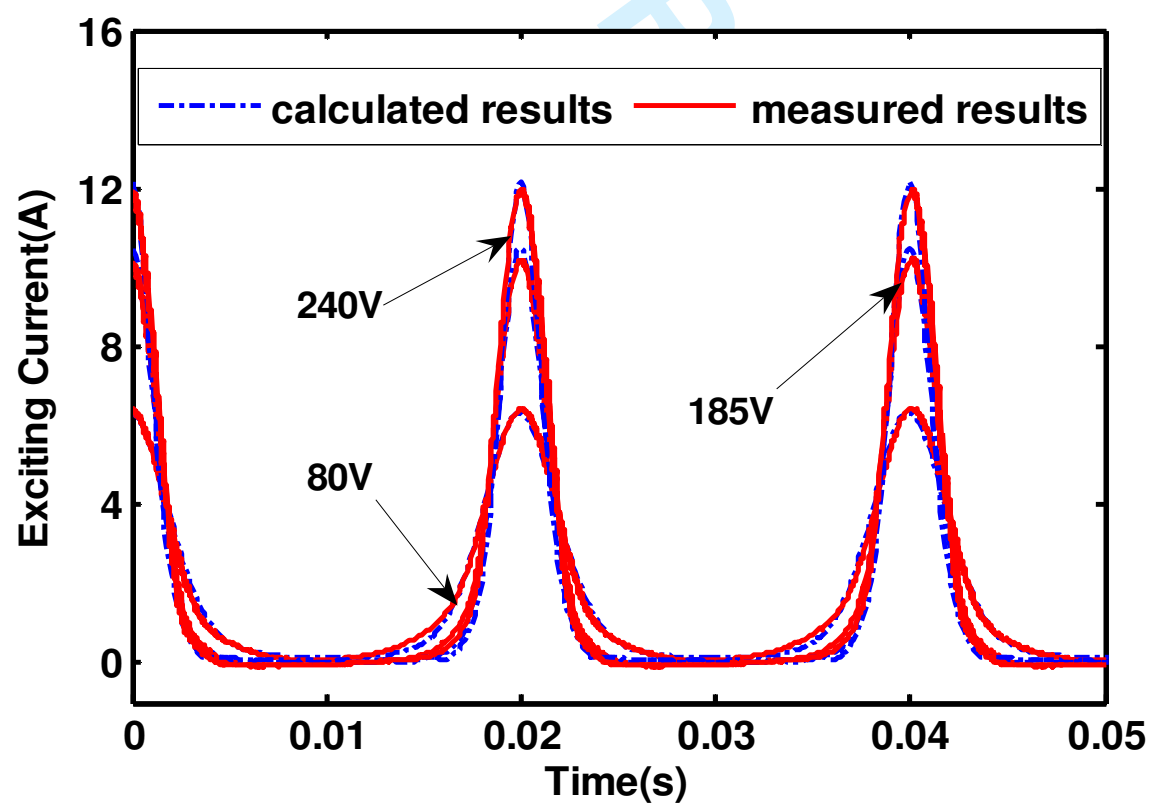

(b) $I_{d c}=1.69 \mathrm{~A} ; U_{a c}=80 \mathrm{~V}, 185 \mathrm{~V}, 240 \mathrm{~V}$

Figure 3. Exciting currents under different DC bias conditions

The truncated harmonic number $N$ should be properly selected considering the DC and AC excitations. In this paper at least 9 harmonics are truncated in the computation to control the errors of exciting current within 5\%. From Table II it can be concluded that the iterative number for 
convergence depends on the DC bias excitation, truncated harmonic number $N(N=9)$ and convergence criterion $\left(\varepsilon_{1}=\varepsilon_{2}=0.005\right)$.

\section{Analysis of the flux density and flux distribution under DC bias condition}

One point on the cross section of the LCM shown in Figure 4, such as point B, can be selected to observe the effect of DC bias on the total flux. The flux density $B_{x}$ in point $\mathrm{B}$ is calculated by the decomposed HBFEM and the time domain finite element method (TDFEM) respectively $\left(B_{y}\right.$ is too small to be analyzed). The waveforms of $B_{x}$ shown in Figure 5 demonstrate that the DC-biasing magnetic fields calculated by the two methods coincide well. Due to the invade of DC bias, the total flux is raised by the DC flux and saturates the magnetic core in one direction, which corresponds to the exciting current distorted in half-cycle shown in Figure 3. Table III presents the corresponding harmonic components of $B_{x}$ in Figure 5.

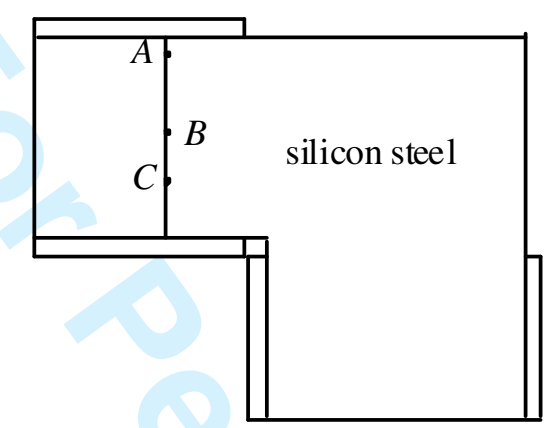

Figure 4. Computational region: one quarter of the square laminated core model

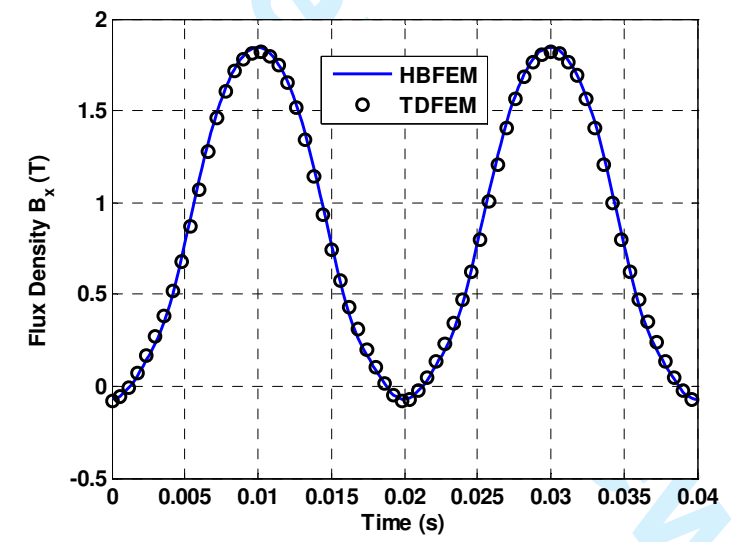

Figure 5. Flux density $B_{x}$ in point B $\quad\left(I_{d c}=1.27 \mathrm{~A}, U_{a c}=370 \mathrm{~V}\right)$

TABLE III

Harmonic components of $B_{x}$ in point B

\begin{tabular}{ccccccccccc}
\hline \hline $\begin{array}{c}\text { Harmonic } \\
\text { components }\end{array}$ & $B_{\mathrm{x} 0}$ & $B_{\mathrm{x} 1}$ & $B_{\mathrm{x} 2}$ & $B_{\mathrm{x} 3}$ & $B_{\mathrm{x} 4}$ & $B_{\mathrm{x} 5}$ & $B_{\mathrm{x} 6}$ & $B_{\mathrm{x} 7}$ & $B_{\mathrm{x} 8}$ & $B_{\mathrm{x} 9}$ \\
Flux & & & & & & & & & & \\
density (T) & 0.9256 & 0.9847 & 0.0509 & 0.0299 & 0.0122 & 0.0016 & 0.0045 & 0.0015 & 0.0019 & 0.0013 \\
\hline \hline
\end{tabular}

Note: $B_{x 0}=\left|B_{x, 0}\right|$ and $B_{x i}=\sqrt{B_{x, 2 i-1}^{2}+B_{x, 2 i}^{2}}(i=1,2, \ldots, 9)$.

Actually the DC flux is not only affected nonlinearly by the DC bias, but also depends on the alternating voltage. Meanwhile the AC flux varies with different DC bias conditions. Harmonic 
components of $B_{x}$ in point B is observed under different DC bias conditions. As Table III shows, the DC and fundamental components are dominant in flux density $B_{x}$. Therefore five different DC bias levels and eight different AC excitation cases shown in Figure 6 and Figure 7 are integrated to analyze the variation of the DC component $B_{x 0}$ and the fundamental component $B_{x 1}$ under DC bias excitation.

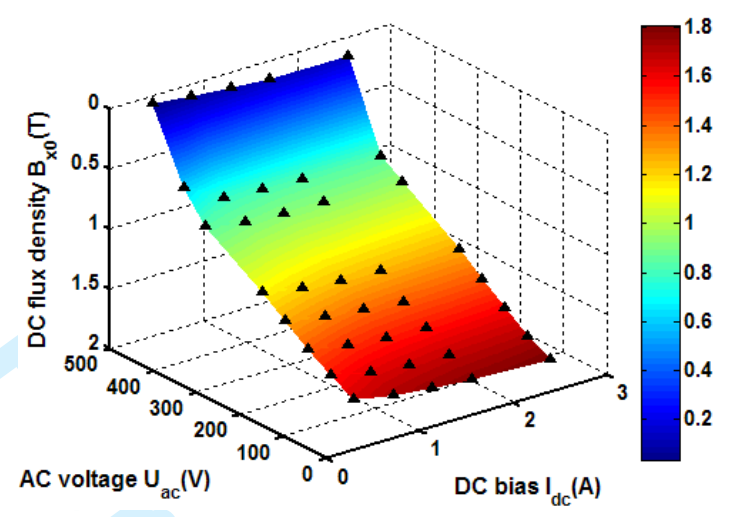

Figure 6. DC flux density $\left(B_{\mathrm{x} 0}\right)$ under different DC bias and AC excitations

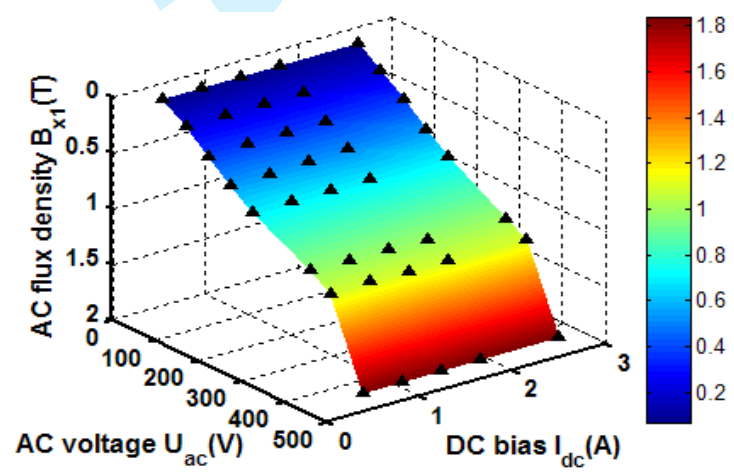

Figure 7. AC flux density $\left(B_{\mathrm{x} 1}\right)$ under different DC bias and AC excitations

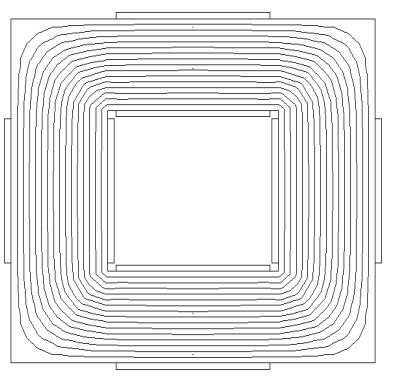

a. DC flux

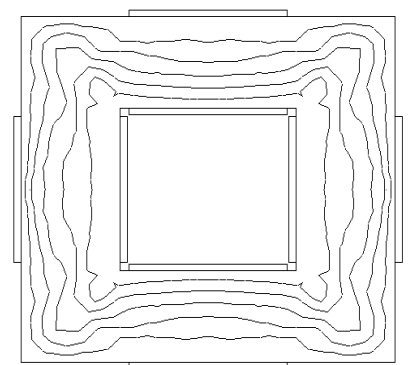

d. third order $(3 \omega \mathrm{t}=\pi / 2)$

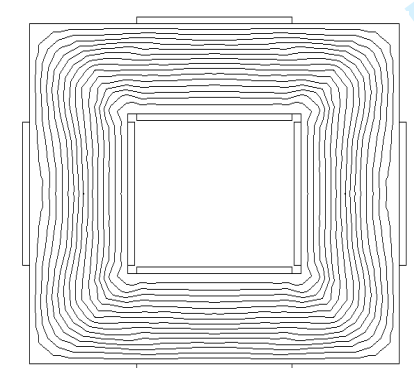

b. fundamental $(\omega \mathrm{t}=\pi / 2)$

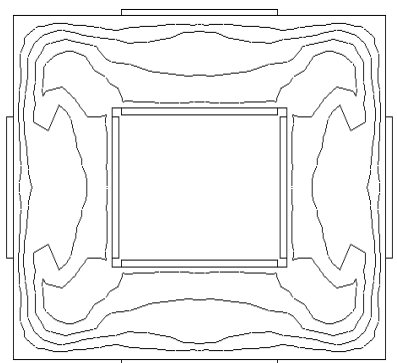

e. fourth order $(4 \omega \mathrm{t}=\pi / 2)$

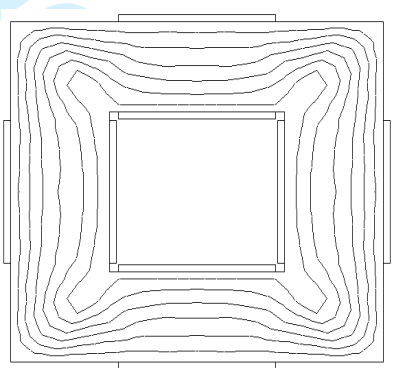

c. second order $(2 \omega \mathrm{t}=\pi / 2)$

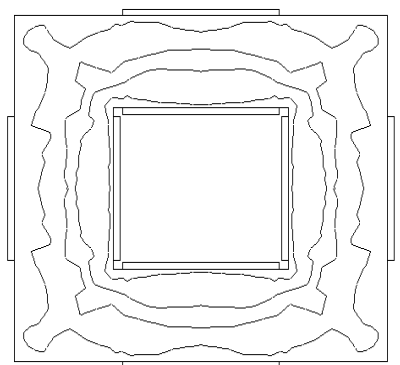

f. fifth order $(5 \omega t=\pi / 2)$ 
Figure 8. Harmonic flux distributions $\left(I_{d c}=1.27 \mathrm{~A}, U_{a c}=370 \mathrm{~V}\right)$

Figure 6 shows that the DC flux density increases slowly and tends to be constant with the increment of DC bias when the alternating voltage is kept constant. When the DC bias is kept constant, the AC excitation will suppress the DC flux density nonlinearly. It also can be concluded from Figure 7 that the DC bias almost has no influence on the AC flux density, whereas the AC excitation gives rise to the variation of AC flux density.

Harmonic flux distributions under DC bias condition are presented in Figure 8. The harmonic flux distributions vary with the time (related to phase angle) and excitations (related to DC bias and alternating voltage). The high-order harmonic distribution exhibits the characteristics of disorder under DC bias condition. It is indicated that the disorder in harmonic flux distributions may affect and distorted the total flux, which is the superposition of all harmonic components.

\section{Conclusion}

The decomposed HBFEM combined with the Gauss-Seidel iterative scheme is presented to investigate the DC bias phenomena in saturated electromagnetic devices. The harmonic solutions are solved separately and sequentially considering electromagnetic coupling. The presented method and the corresponding solution scheme are applicable to large-scale computation of DC-biasing magnetic field.

The DC flux raises the total flux and saturates the ferromagnetic core rapidly and significantly in one direction. The exciting current is distorted seriously as a result of a large spectrum of harmonics. Harmonic flux distributions under DC bias conditions reflect the incidence of saturation of ferromagnetic core.

\section{References}

Bachinger, F., Langer, U. and Schoberl, J. (2006), "Efficient solvers for nonlinear time-periodic eddy current problems", Comput. Visual. Sci., Vol 9 No 4, pp: 197-207.

Biro, O., Buchgraber, G., Leber, G. and Preis, K. (2008), "Prediction of magnetizing current waver-formers in a three-phase power transformer under DC bias", IEEE Trans. on Mag., Vol. 44 No. 6, pp. 1554-7.

Bolduc, L. (2002), "GIC observations and studies in the Hydro-Quebec power system", Journal of Atmospheric and Solar-Terrestrial physics, 64, 1793-802.

Cheng, Z., Norio, T. and Behzad, F. (2009), Electromagnetic and Thermal Field Modeling and Application in Electrical Engineering, Science Press, Beijing, China.

Deblecker, O. and Lobry, J. (2006), "A new efficient technique for harmonic-banlance finite-element analysis of saturated electromagnetic devices", IEEE Trans. on Mag., Vol. 42 No. 4, pp. 535-538.

Gyselinck, J., Dular, P., Geuzaine, C. and Legros, W. (2002), "Harmonic-balance finite-element modeling of electromagnetic devices: A novel approach”, IEEE Trans. on Mag., Vol. 38 No. 2, pp. 521-4.

Lombard, P. and Meunier G. (1992), "General method for electric and magnetic coupled problem in 2D and magnetodynamic domain", IEEE Trans. on Mag., Vol. 28 No. 2, pp. 1291-4.

Lu, J., Yamada, S. and Harrison H.B. (1996), "Application of harmonic balance-finite element 
method (HBFEM) in the design of switching power supplies", IEEE Trans. on Power Electronics, Vol. 11 No. 2, pp. 347-55.

Picher, P., Bolduc, L., Dutil, A. and Pham, V.Q. (1997), "Study of the acceptable DC current limit in core-form power transformers", IEEE Trans. on Power Delivery, Vol. 12 No. 1, pp. 257-65.

Pirjola, R. (2000), "Geomagnetically induced current during magnetic storms", IEEE Trans. Power Apparatus and Systems, Vol. 104 No. 1, pp. 212-219.

Saad, Y. (2009), Iterative methods for sparse linear systems, Science Press, Beijing, China.

Tay, H.C. and Swift, G.W. (1985), "on the problem of transformer overheating due to geomagnetically induced current”, IEEE Trans. Power Apparatus and Systems, Vol. 104 No. 1, pp. 212-219.

Yamada, S., Biringer, P. and Bessho, K. (1991), "Calculation of nonlinear eddy-current problems by the harmonic balance finite element method”, IEEE Trans. on Mag., Vol. 27 No. 5, pp. $4122-5$.

Yamada, S. and Bessho, K. (1988), "Harmonic field calculation by the combination of finite element analysis and harmonic balance method", IEEE Trans. on Mag., Vol. 24 No. 6, pp. 2588-90.

Yamada, S., Bessho, K. and Junwei, L. (1989), "Harmonic balance finite element method applied to nonlinear AC magnetic analysis", IEEE Trans. on Mag., Vol. 25 No. 4, pp. 2971-3.

Yamada, S., Biringer, P., Hirano, K. and Bessho, K. (1990), "Finite element analysis of nonlinear dynamic magnetic field with DC component in the harmonic domain", IEEE Trans. on Mag., Vol. 26 No. 5, pp. 2199-201.

Yao, Y., Koh, C.S., Ni, G. and Xie, D. (2005), “3-D nonlinear transient eddy current calculation of noline power transformer under DC bias”, IEEE Trans. on Mag., Vol. 41 No. 5, pp. 1840-3.

You, Y., Fuchs, E.F., Lin, D. and Barnes, P.R. (1996), "Reactive power demand of transformers with DC bias", IEEE Industry Application Magazine, Vol. 2 No. 4, pp. 45-52.

Zhang, B., Cui, X., Zeng, R. and He, J. (2006), "Calculation of DC current distribution in AC power system near HVDC system by using moment method coupled to circuit equations", IEEE Trans. on Mag., Vol. 42 No. 4, pp. 703-6.

Zhao, X., Lu, J., Li, L., Cheng, Z. and Lu, T. (2010), “Analysis of the DC bias phenomenon by the harmonic balance finite element method", IEEE Trans. on Power Delivery, accepted. 


\title{
Analysis of Saturated Electromagnetic Devices Under DC Bias Condition by the Decomposed Harmonic Balance Finite Element Method
}

\begin{abstract}
Purpose - This paper aims to introduce the decomposed harmonic balance finite element method (HBFEM) to decrease the memory requirement in large-scale computation of the DC-biasing magnetic field. Harmonic analysis of the flux density and flux distribution was carried out to investigate the DC biased problem in a laminated core model (LCM).

Design/methodology/approach - Based on the DC bias test on a LCM, the decomposed HBFEM is applied to accurately calculate the DC-biasing magnetic field. External electric circuits are coupled with the magnetic field in the harmonic domain. The reluctivity matrix is decomposed and the block Gauss-Seidel algorithm solves each harmonic solution of magnetic field and exciting current sequentially.

Findings - The calculated exciting currents and flux density are compared with that obtained from measurement and time domain finite element analysis respectively, which demonstrates consistency. The DC bias leads to the significant saturation of the magnetic core and serious distortion of the exciting current. The flux density varies nonlinearly with DC bias excitation.

Research limitations/implications - The harmonic balance method is only applicable in solving the steady state magnetic field. Future improvements in the method are necessary in order to manage the hysteresis effects in magnetic material.

Originality/value - The proposed method to solve the DC biased problem significantly reduces the memory requirement compared to the conventional HBFEM. The decomposed harmonic balance equations are solved efficiently by the block Gauss-Seidel algorithm combined with the relaxation iterative scheme. An investigation on DC bias phenomena is carried out through the harmonic solution of the magnetic field. The decomposed HBFEM can also be applied to solve 3-D DC-biasing magnetic field and eddy current nonlinear problems in a practical power transformer.
\end{abstract}

Keywords - Harmonic balance, LCM, DC bias, flux density

\section{Introduction}

Electromagnetic devices such as electrical machines and power transformers are generally operated under steady-state conditions. Owing to the nonlinearity of magnetic material in electromagnetic devices, there are often high-order harmonics in the exciting current and magnetic field. The HBFEM has been proposed to solve the steady-state magnetic field with eddy current problems (Yamada et al., 1988; Yamada et al. 1989; Bachinger et al., 2006) and to design the switching power supplies (Lu et al., 1996). A modified method via block decomposition of the system equation has been presented to reduce the memory requirement (Yamada et al., 1991), although both the DC component and harmonics are not considered in computation. Based on the harmonic balance technique, a generalized parametric formulation of the nonlinearity and the Jiles-Atherton hysteresis model has been employed to analyze the saturating and hysteresis characteristics of the inductor (Rizzoli et al., 1995). The HBFEM has been further developed by introducing differential reluctivity tensor (Gyselinck et al., 2002) and transmission-line modeling techniques (Deblecker et al., 2006) respectively. The magnetic field of a three phase transformer 
was also calculated. The fixed-point technique has been introduced to calculate the nonlinear eddy current problems stepping through one period only and allows all harmonics to be solved in parallel (Biro et al., 2006; Ausserhofer et al., 2007; Ciric et al., 2007). The DC biased problem of power transformers has been paid more attention in recent years (Picher et al., 1997; Yao et al., 2005; Biro et al., 2008). The magnetic storm and HVDC transmission system may generate large quasi-direct and direct current respectively on the earth (Pirjola et al., 2000; Zhang et al., 2006). Harmonic analysis of exciting current and flux density has been done by the conventional HBFEM to investigate the DC bias phenomena (Zhao et al., 2010). However, the conventional approach generates a large-size system matrix to solve all harmonic solutions simultaneously. As a result of the large memory requirement the widespread application of conventional HBFEM has been limited in large-scale computation.

This paper introduces the decomposed HBFEM to calculate the DC-biasing magnetic field of LCM in the harmonic domain, considering the harmonic coupling between the external circuits and magnetic field. The magnetic reluctivity matrix can be decomposed in the harmonic domain, thus each harmonic solution is solved sequentially. A modified resolution scheme for the nonlinear equation set is developed at the same time. A more detailed analysis of the flux density and flux distribution under different DC bias conditions is carried out through the harmonic solutions of the magnetic field.

\section{Decomposed harmonic balanced system equation}

The following vector potential equation can be used to describe the two dimensional magnetic field, where $A$ and $J$ are magnetic vector potential and current density respectively, $v$ is the magnetic reluctivity, and $\sigma$ is the conductivity.

$$
\nabla \times(\nu \nabla \times A)+\sigma(\partial A / \partial t)=J .
$$

Due to the periodic characteristics of the electromagnetic field under DC bias conditions, the steady state variables such as exciting current density $J$, magnetic vector potential $A$, and flux density $B$ can be approximated by a summation of trigonometric function as follows:

$$
P(t)=P_{0}+\sum_{i=1}^{\infty}\left(P_{2 i-1} \sin i \omega t+P_{2 i} \cos i \omega t\right)
$$

where $P(t)$ can be replaced by $J, A, B_{\mathrm{x}}$ and $B_{\mathrm{y}}$. The symbols $i$ and $\omega$ represent the harmonic number and fundamental angular frequency respectively.

The magnetic reluctivity $v$, which is a function of magnetic flux density $B$, can also be expressed by:

$$
v(t)=H(t) / B(t)=v_{0}+\sum_{i=1}^{\infty}\left(v_{2 i-1} \sin i \omega t+v_{2 i} \cos i \omega t\right) .
$$

The Galerkin's method and finite element method can be applied to discretize the governing equation for two dimensional problems. Equation (1) is written as:

$$
\iint_{\Omega_{e}}\left(\frac{\partial N_{m}}{\partial x} \nu \frac{\partial A}{\partial x}+\frac{\partial N_{m}}{\partial y} \nu \frac{\partial A}{\partial y}\right) d x d y+\iint_{\Omega_{e}}\left(\sigma \frac{\partial A}{\partial x}\right) d x d y=\iint_{\Omega_{e}}\left(J \cdot N_{m}\right) d x d y
$$

where $N_{\mathrm{m}}$ is the interpolation functions on node $m$ in the finite element region $\Omega_{\mathrm{e}}$.

The decomposed finite element equation can be obtained by substituting periodic variables expressed in (2) into equation (4) and equating the coefficients of $\sin (i \omega t)$ and $\cos (i \omega t)$ on both 
sides according to the harmonic balance method:

$$
\begin{aligned}
& \left(\boldsymbol{S}^{e} * \boldsymbol{R}_{i, i}^{e}+\boldsymbol{T}^{e} * \boldsymbol{h}_{i}\right) \boldsymbol{A}_{i}^{e}=-\sum_{j=1,2,3 \ldots}^{j \neq i}\left(\boldsymbol{S}^{e} * \Re_{i, j}^{e}\right) \boldsymbol{A}_{j}^{e}+\boldsymbol{K}_{i}^{e} \quad(i=1,2 \ldots N) \\
& \boldsymbol{A}_{i}^{e}=\left\{\begin{array}{l}
\left\{A_{0}^{1}, A_{1}^{1}, A_{2}^{1}, A_{0}^{2}, A_{1}^{2}, A_{2}^{2}, \cdots, A_{0}^{q}, A_{1}^{q}, A_{2}^{q}\right\}^{T} \quad(i=1) \\
\left\{A_{i}^{1}, A_{i+1}^{1}, A_{i}^{2}, A_{i+1}^{2}, \cdots, A_{i}^{q}, A_{i+1}^{q}\right\}^{T} \quad(i=2, \ldots N)
\end{array},\right. \\
& \boldsymbol{K}_{i}^{e}=\iint_{\Omega^{e}}\left(\boldsymbol{J}^{e} \cdot N_{m}\right) d x d y \quad(m=1,2, \cdots q) \\
& =\left\{\begin{array}{l}
\left\{K_{0}^{1}, K_{1}^{1}, K_{2}^{1}, K_{0}^{2}, K_{1}^{2}, K_{2}^{2}, \cdots, K_{0}^{q}, K_{1}^{q}, K_{2}^{q}\right\}^{T} \quad(i=1) \\
\left\{K_{i}^{1}, K_{i+1}^{1}, K_{i}^{2}, K_{i+1}^{2}, \cdots, K_{i}^{q}, K_{i+1}^{q}\right\}^{T} \quad(i=2, \ldots N)
\end{array},\right.
\end{aligned}
$$

where $N$ is the truncated harmonic number and $q$ is the total node number in an element. $\boldsymbol{J}^{\mathrm{e}}$ has the same vector form with $\boldsymbol{A}_{i}^{e} . \boldsymbol{K}_{i}$ is obtained from the spatial distribution of the $i$-th harmonic component of current density.

The operator $*$ in equation (5) can be defined by:

$$
\boldsymbol{S}^{e} * \boldsymbol{R}_{i, i}^{e}+\boldsymbol{T}^{e} * \boldsymbol{h}_{i}=\left[\begin{array}{ccc}
S_{11} \mathfrak{R}_{i, i}^{e} & S_{12} \boldsymbol{R}_{i, i}^{e} & \cdots \\
S_{21} \Re_{i, i}^{e} & S_{22} \mathfrak{R}_{i, i}^{e} & \cdots \\
\vdots & \vdots & \ddots
\end{array}\right]+\left[\begin{array}{ccc}
T_{11} \boldsymbol{h}_{i} & T_{12} \boldsymbol{h}_{i} & \cdots \\
T_{21} \boldsymbol{h}_{i} & T_{22} \boldsymbol{h}_{i} & \cdots \\
\vdots & \vdots & \ddots
\end{array}\right],
$$

where $S_{m, n}$ and $T_{m, n}$ are the elements in $\boldsymbol{S}^{e}$ and $\boldsymbol{T}^{e}$ respectively. The two terms can be defined by the following expressions:

$$
\begin{aligned}
& S_{m, n}=\int_{\Omega_{e}} \nabla N_{m} \cdot \nabla N_{n} d \Omega, \\
& T_{m, n}=\int_{\Omega_{e}} \sigma N_{m} \cdot N_{n} d \Omega,
\end{aligned}
$$

where the subscripts $m$ and $n$ represent the node number in an element $(m, n=1,2, \ldots q)$.

The matrices $\mathfrak{R}_{i, i}^{e}$ and $\mathfrak{R}_{i, j}^{e}$ are determined by the Fourier coefficients in equation (3). They act as a reluctivity and are called the reluctivity matrix, which is derived from the multiplication of magnetic vector potential $A$ in equation (2) and reluctivity $v$ in equation (3) (Yamada et al., 1991). The DC component of the magnetic vector potential is coupled with the fundamental harmonic in computation, therefore the expression of matrix $\mathfrak{R}_{i, i}^{e}(i=1)$ differs from $\mathfrak{R}_{i, i}^{e}(i>1)$ shown in equation (11) and matrix $\mathfrak{R}_{i, j}^{e}$, which is expressed by equations (12), (13) and (14):

$$
\begin{aligned}
& \mathfrak{R}_{i, i}^{e}=\left\{\begin{array}{l}
\frac{1}{2}\left[\begin{array}{ccc}
2 v_{0} & v_{1} & v_{2} \\
2 v_{1} & 2 v_{0}-v_{4} & v_{3} \\
2 v_{2} & v_{3} & 2 v_{0}+v_{4}
\end{array}\right](i=1), \\
\frac{1}{2}\left[\begin{array}{cc}
2 v_{0}-v_{4 i} & v_{4 i-1} \\
v_{4 i-1} & 2 v_{0}+v_{4 i}
\end{array}\right] \quad(i>1)
\end{array}\right. \\
& \boldsymbol{R}_{1, i}^{e}=\frac{1}{2}\left[\begin{array}{cc}
v_{2 i-1} & v_{2 i} \\
v_{2 i-2}-v_{2 i+2} & v_{2 i+1}-v_{2 i-3} \\
v_{2 i+1}+v_{2 i-3} & v_{2 i-2}+v_{2 i+2}
\end{array}\right](i>1),
\end{aligned}
$$




$$
\begin{gathered}
\left(\boldsymbol{R}_{i, 1}^{e}\right)^{T}=\frac{1}{2}\left[\begin{array}{cc}
2 v_{2 i-1} & 2 v_{2 i} \\
v_{2 i-2}-v_{2 i+2} & v_{2 i+1}-v_{2 i-3} \\
v_{2 i+1}+v_{2 i-3} & v_{2 i-2}+v_{2 i+2}
\end{array}\right](i>1), \\
\Re_{i, j}^{e}=\left(\boldsymbol{R}_{j, i}^{e}\right)^{T}=\frac{1}{2}\left[\begin{array}{cc}
v_{2\left(m^{\prime}-n^{\prime}\right)}-v_{2\left(m^{\prime}+n^{\prime}\right)} & v_{2\left(m^{\prime}-n^{\prime}\right)-1}+v_{2\left(m^{\prime}-n^{\prime}\right)+1} \\
v_{2\left(m^{\prime}-n^{\prime}\right)+1}-v_{2\left(m^{\prime}-n^{\prime}\right)-1} & v_{2\left(m^{\prime}-n^{\prime}\right)}+v_{2\left(m^{\prime}+n^{\prime}\right)}
\end{array}\right](i, j>1 \& i \neq j),
\end{gathered}
$$

where the subscripts $m^{\prime}$ and $n^{\prime}$ are relevant to the harmonic number $i$ and $j(i, j>1$ and $i \neq j) . m^{\prime}$ is defined by $\operatorname{Max}(i, j)$ and $n^{\prime}$ by $\operatorname{Min}(i, j)$.

The matrix $\boldsymbol{h}_{i}$ is a constant concerned with harmonic orders and is called the harmonic matrix. It is presented as follows:

$$
\boldsymbol{h}_{i}=\left\{\begin{array}{l}
\omega\left[\begin{array}{ccc}
0 & 0 & 0 \\
0 & 0 & -1 \\
0 & 1 & 0
\end{array}\right](i=1) \\
\omega\left[\begin{array}{cc}
0 & -i \\
i & 0
\end{array}\right] \quad(i>1)
\end{array} .\right.
$$

By assembling all finite elements in the computational region, equations in (5) can be written as:

$$
\boldsymbol{Q}_{i} \boldsymbol{A}_{i}=\boldsymbol{K}_{i}+\boldsymbol{F}_{i} \quad(i=1,2, \ldots, N)
$$

where $\boldsymbol{F}_{i}=-\sum_{j=1,2,3 \ldots}^{j \neq i}\left(\boldsymbol{S} * \boldsymbol{R}_{i, j}\right) \boldsymbol{A}_{j}$ and $\boldsymbol{Q}_{i}=\boldsymbol{S} * \boldsymbol{R}_{i, i}+\boldsymbol{T} * \boldsymbol{h}_{i}$

\section{Magnetic field coupled with electric circuits}

When electromagnetic devices are excited by voltage sources, the electric potential difference in the coil region can be obtained from the following equation (Lombard et al., 1992):

$$
U_{k}=R_{k} I_{k}+L_{k} \frac{d I_{k}}{d t}+\frac{1}{C_{k}} \int I_{k} d t+\frac{N_{k} l_{k}}{S_{k}} \int_{\Omega_{c}}(\partial A / \partial t) d \Omega,
$$

where $U_{k}$ is the input voltage of circuit $k$, and $I_{k}$ is the corresponding exciting current. $R_{k}$ and $S_{k}$ represent the resistance and cross-sectional area of the $k$-th winding respectively. $L_{k}$ is the inductance and $C_{k}$ is the capacitance of the external circuit $k . N_{k}$ is the turn number of the $k$-th winding, and $l_{k}$ is the thin wire's length in the $\mathrm{z}$-direction. In the DC-biasing case the input voltage can be written as follows:

$$
U_{k}=U_{k, 0}+\sum_{i=1}^{\infty}\left(U_{k, 2 i-1} \sin i \omega t+U_{k, 2 i} \cos i \omega t\right) .
$$

Hence the harmonics in voltages, currents and magnetic vector potentials can be expressed separately by the following equation:

$$
\begin{gathered}
\boldsymbol{U}_{k, i}=\boldsymbol{Z}_{k, i} \boldsymbol{J}_{k, i} S_{k}+\boldsymbol{C}_{k, i} \boldsymbol{A}_{i} \\
\boldsymbol{C}_{k, i}=\sum_{n=1}^{N_{c}} \frac{\omega N_{k} l_{k}}{3 S_{k}}\left[\begin{array}{lll}
\boldsymbol{h}_{i} & \boldsymbol{h}_{i} & \boldsymbol{h}_{i}
\end{array}\right]
\end{gathered}
$$




$$
\boldsymbol{Z}_{k, i}=\left[\begin{array}{cc}
R_{k}+i \omega L_{k}-\left(i \omega C_{k}\right)^{-1} & 0 \\
0 & R_{k}-i \omega L_{k}+\left(i \omega C_{k}\right)^{-1}
\end{array}\right]
$$

where the subscripts $i$ indicate the harmonic number. $\boldsymbol{U}_{k, i}$ and $\boldsymbol{J}_{k, i}$ are the $i$-th harmonic vectors of the input voltage and the current density in circuit $k$, respectively. $\boldsymbol{C}_{k, i}$ is the coupled matrix linking the electric circuit $k$ with the magnetic field. Nonlinear components in external circuits can be expressed by harmonic impedance matrices $\boldsymbol{Z}_{k, i} . N_{c}$ is the finite element number in the coil region.

The harmonic solutions of magnetizing currents and magnetic vector potentials are obtained by solving equations (16) and (19) together, which is shown by the following equation:

$$
\left[\begin{array}{cc}
\boldsymbol{Q}_{i} & \boldsymbol{G}_{k, i} \\
\boldsymbol{C}_{k, i} & \boldsymbol{Z}_{k, i}
\end{array}\right]\left\{\begin{array}{c}
\boldsymbol{A}_{i} \\
\boldsymbol{J}_{k, i}
\end{array}\right\}=\left\{\begin{array}{c}
\boldsymbol{F}_{i} \\
\boldsymbol{U}_{k, i}
\end{array}\right\}(i=1,2, \ldots, N)
$$

where the matrix $\boldsymbol{G}_{k, i}$ is related to the spatial distribution of the $i$-th harmonic component of the current density. Finally, $N$ separated equations shown in (22) constitute the system equation when $N$ harmonics are considered in the computation.

\section{Computational procedure based on the block Gauss-Seidel algorithm}

\subsection{Previous calculation procedure}

In Yamada et al. (1991), a calculation procedure to solve the equation (16) has been proposed:

1) First, the fundamental harmonic $\boldsymbol{A}_{1}$ is calculated based on the assumption of setting $\boldsymbol{A}_{2} \sim \boldsymbol{A}_{N}$ to zero. If the convergence of $\boldsymbol{A}_{1}$ is satisfied, turn to (2). If not, update $\boldsymbol{A}_{1}$ and repeat (1).

2) The other harmonics, $\boldsymbol{A}_{2} \sim \boldsymbol{A}_{N}$, are calculated respectively with the known $\boldsymbol{A}_{1}$. If the convergence of $\boldsymbol{A}_{2} \sim \boldsymbol{A}_{N}$ is satisfied, the calculation procedure stops. If not, turn to (1).

However, the computational procedure above is not dependable and efficient. The convergent criterion in each harmonic computation and the lack of full use of updated solutions in nonlinear iterations leads to convergence uncertainty and the subsequent inaccuracy of harmonic solutions, especially when the nonlinearities of the magnetic material are strong. Yamada et al (1991) also pointed out that there is convergence uncertainty in the iterative approach. In fact, a compulsory stop is usually done by setting a maximum number of iterative steps.

\subsection{Improved computational procedure}

The nonlinear terms $\boldsymbol{Q}_{i}$ in equation (16) are obtained from $\boldsymbol{R}_{i, i}$ in equation (11). The diagonal elements in matrices $\Re_{i, i}$ and $S$ in equation (8) are dominant. Therefore, a diagonally dominant characteristic exists in the system equation (22) and the block Gauss-Seidel algorithm (Saad et al., 2009) can be used to solve the DC-biasing magnetic field. The new computational procedure is as follows:

1) ( $p=0)$ : Initialize $\boldsymbol{A}_{1}^{0}, \boldsymbol{A}_{2}^{0} \ldots \boldsymbol{A}_{\mathrm{N}}^{0}$ and solve the decomposed harmonic balance equation set in (27) following the procedure at step (2).

2) $(p>0)$ :

a). $(i=1)$ : Update $\boldsymbol{Q}_{1}^{\mathrm{p}}$ by $\boldsymbol{A}_{1}^{\mathrm{p}}, \boldsymbol{A}_{2}^{\mathrm{p}}, \ldots \boldsymbol{A}_{\mathrm{N}}^{\mathrm{p}}$ and $\boldsymbol{F}_{1}^{\mathrm{p}}$ by $\boldsymbol{A}_{2}^{\mathrm{p}}, \boldsymbol{A}_{3}^{\mathrm{p}}, \ldots \boldsymbol{A}_{\mathrm{N}}^{\mathrm{p}}$. Assemble the matrices $\boldsymbol{C}_{1}$ and $\boldsymbol{G}_{1}$. Solve the equations (27) to obtain the renewed fundamental harmonic vector $\boldsymbol{A}_{1}^{\mathrm{p}+1}$.

b). $(1<i<N)$ : Update $\boldsymbol{Q}_{\mathrm{i}}^{\mathrm{p}}$ by $\boldsymbol{A}_{1}^{\mathrm{p}}, \ldots \boldsymbol{A}_{\mathrm{i}}^{\mathrm{p}}, \ldots \boldsymbol{A}_{\mathrm{N}}^{\mathrm{p}}$ and $\boldsymbol{F}_{\mathrm{i}}^{\mathrm{p}}$ by $\boldsymbol{A}_{1}^{\mathrm{p}+1}, \boldsymbol{A}_{2}^{\mathrm{p}+1}, \ldots \boldsymbol{A}_{\mathrm{i}-1}^{\mathrm{p}+1}, \boldsymbol{A}_{\mathrm{i}+1}^{\mathrm{p}}, \ldots \boldsymbol{A}_{\mathrm{N}}^{\mathrm{p}}$. Assemble 
the matrices $\boldsymbol{C}_{\mathrm{i}}$ and $\boldsymbol{G}_{\mathrm{i}}$. Solve the $i$-th equation in (27) to obtain the renewed $i$-th harmonic vector $\boldsymbol{A}_{\mathrm{i}}^{\mathrm{p}+1}$.

c). $(i=N)$ : Update $\boldsymbol{Q}_{\mathrm{N}}^{\mathrm{p}}$ by $\boldsymbol{A}_{1}^{\mathrm{p}}, \ldots \boldsymbol{A}_{\mathrm{N}-1}^{\mathrm{p}}, \boldsymbol{A}_{\mathrm{N}}^{\mathrm{p}}$ and $\boldsymbol{F}_{\mathrm{N}}^{\mathrm{p}}$ by $\boldsymbol{A}_{1}^{\mathrm{p}+1}, \boldsymbol{A}_{2}^{\mathrm{p}+1}, \ldots \boldsymbol{A}_{\mathrm{N}-1}^{\mathrm{p}+1}$. Assemble the matrices $\boldsymbol{C}_{\mathrm{N}}$ and $\boldsymbol{G}_{\mathrm{N}}$. Solve the $N$-th equation in (27) to obtain the renewed $N$-th harmonic vector $\boldsymbol{A}_{\mathrm{N}}^{\mathrm{p}+1}$.

3) Check for convergence criterion. If satisfied the calculation stops, otherwise renew all harmonic solutions with a properly selected relaxation factor, $A^{\text {new }}=(1-\alpha) A^{\text {now }}+\alpha A^{\text {old }}$, and return to step (2) for the next iteration $(p=p+1)$.

The symbol $i$ refers to the harmonic number in the computation. $N$ is the truncated harmonic number and $p$ represents the number of iterative steps. $\varepsilon$ is an imposed tolerance. The symbol $\alpha$ is a properly selected relaxation factor aimed at rapid convergence of the harmonic solution in nonlinear iterations. The stopping criterion is defined by:

$$
\frac{\left\|\boldsymbol{X}^{p+1}-\boldsymbol{X}^{p}\right\|}{\left\|\boldsymbol{X}^{p}\right\|}<\varepsilon
$$

where $X$ represents all harmonic solutions that correspond to equation set (22), including magnetic vector potential $\boldsymbol{A}$ and current density $\boldsymbol{J}$.

\subsection{Comparison between the previous method and the new method}

Compared with the previous calculation procedure in 4.1 , the improved computational approach in 4.2 makes the harmonic solutions converge smoothly and unconditionally. The Gauss-Seidel algorithm can be used to solve the nonlinear magnetic field equations more efficiently.

When the DC-biasing magnetic field is calculated, suppose that there are $M_{\mathrm{T}}$ nodes in the finite element region, thus comparison of the memory required in the computation can be indicated by the largest matrix size as follows:

$$
\frac{M S_{N}}{M S_{T}}=\frac{\left[3 \times M_{T}\right]^{2}}{\left[(2 N+1) \times M_{T}\right]^{2}}=\frac{3 \times 3}{(2 N+1)^{2}},
$$

where $M S_{N}$ represents the matrix size of $\boldsymbol{Q}_{1}$ in equation (22) while $M S_{T}$ represents the system matrix size in traditional HBFEM (Yamada et al., 1990; Zhao et al., 2010). Therefore, the memory requirement has been significantly reduced by the decomposed harmonic balance equations, which are suitable for large-scale computations.

\section{DC-biasing test on the LCM and computational results}

The square LCM shown in Figure 1(a)-(b) was made by the Tianwei Group, Baoding, China. The magnetic core is entwined by two coils of both 312 turns. One is the exciting coil, and the other is the search coil.

\subsection{B-H curve of the LCM}

The ferromagnetic core is laminated by electric sheets, between which there are minor gaps. Therefore, the LCM may have different magnetizing characteristics compared with the steel strips. Some measurements are carried out to obtain the accurate magnetizing curve of LCM. 
The exciting coil is fed by different alternating voltages. The induced voltage $U_{\mathrm{ac}}$ and the exciting current $I$ are measured by the power analyzer, WT-3000, Japan. The experimental data are manipulated as follows:

$$
\begin{aligned}
& \varphi=\left(1 / N_{c o i l}\right) \int U_{a c} d t \\
& B=\varphi / S \\
& \int H \cdot d l=I \cdot N_{c o i l}
\end{aligned}
$$

where $\varphi$ is the magnetic flux in the magnetic core, $N_{\text {coil }}$ is the number of turns of the exciting coil, $S$ is the cross-sectional area of the core, and $l$ is the mean length of the magnetic circuit. The $B-H$ curve [shown in Figure 1(c)] can be determined by solving the equations above.

\subsection{DC-Biasing Test}

The model was tested under different DC bias conditions. The DC bias $I_{d c}$ from a DC current source and alternating voltage from the voltage regulator are applied to the exciting coil. Measurements of no-load induced voltage $\left(U_{a c}\right.$ of $\left.50 \mathrm{~Hz}\right)$ and no-load exciting current have been carried out by the search coil and exciting coil respectively (using the power analyzer WT-3000). In the DC-biasing test the DC bias $I_{d c}$ is smaller than 3 amperes because a DC bias that is too large may damage the LCM. Figure 2 shows the schematic circuit diagram of the DC-biasing test. The electric circuit of the exciting coil is simulated by the harmonic computation. The DC component of voltage $U_{0}$ in equation (18) can be linked with $I_{\mathrm{dc}}$ through the resistance $R$ of the exciting coil.

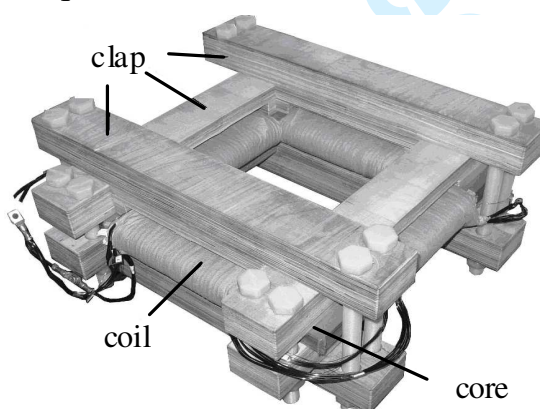

(a) Laminated core model $\left(\sigma_{\text {steel }}=2.22 \times 10^{6} \mathrm{~S} / \mathrm{m}\right)$

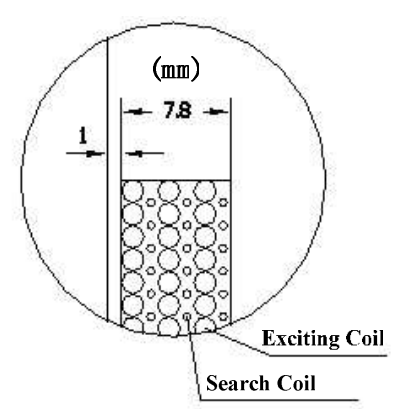

(b) Exciting coil and search coil $\left(\sigma_{\text {coil }}=5.71 \times 10^{7} \mathrm{~S} / \mathrm{m}\right)$

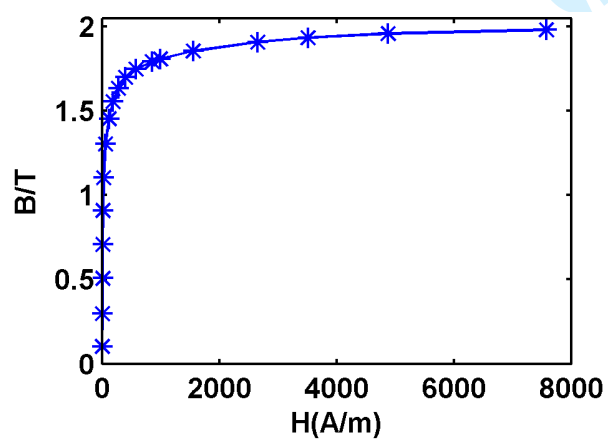

(c) B-H curve of the LCM

Figure 1. LCM and the B-H curve 


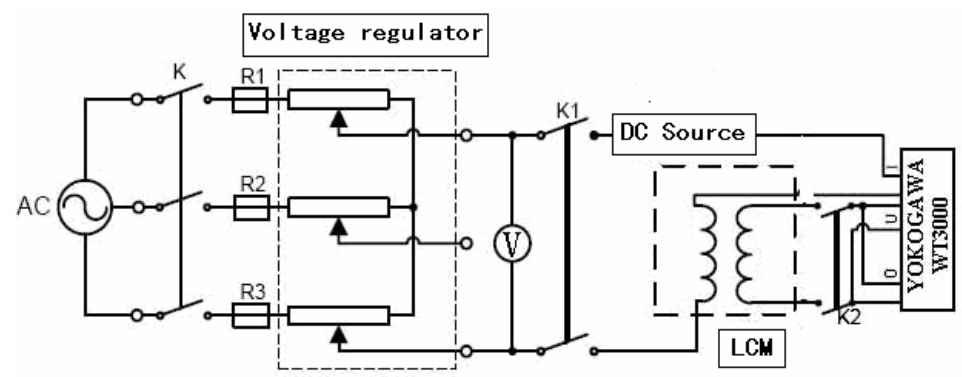

Figure 2. Schematic circuit diagram of the core model in experiment

\subsection{Computational Results}

The exciting currents in the exciting coil and DC-biasing magnetic field are computed simultaneously by the block Gauss-Seidel algorithm proposed in 4.2. The relaxation factor $\alpha$ is initially set to be 0.2 or 0.3 (Cheng et al. 2009) and then varies with the number of iterative steps $P_{c}$. A detailed iterative scheme is shown in Table I. In the harmonic computation 1922 triangular elements and 1014 nodes are used in one quarter of the square model, which is shown in Figure 4.

In Figure 3 below, the calculated exciting currents are compared with the measured current when the truncated harmonic number is selected differently in the computation. The computational results under different DC bias excitations are listed in Table II. $P_{c}$ and $N$ represent the number of iterations for convergence and truncated harmonic number respectively. $I_{r m s, c}$ is the root-mean-square value of calculated exciting current, while $I_{r m s, m}$ is the counterpart obtained from measurement.

Table I Scheme of setting the relaxation factor for the convergence of the harmonic solution

\begin{tabular}{|c|c|c|c|c|c|c|c|c|c|}
\hline$P_{\mathrm{c}}$ & $<10$ & $<20$ & $<40$ & $<60$ & $<70$ & $<80$ & $<90$ & $<100$ & $\geq 100$ \\
\hline$\beta$ & 1 & 0.8 & 0.6 & 0.4 & 0.3 & 0.2 & 0.1 & 0.08 & 0.06 \\
\hline$\alpha=\alpha \cdot \beta$ & 0.2 & 0.16 & 0.12 & 0.08 & 0.06 & 0.04 & 0.02 & 0.016 & 0.012 \\
\hline
\end{tabular}

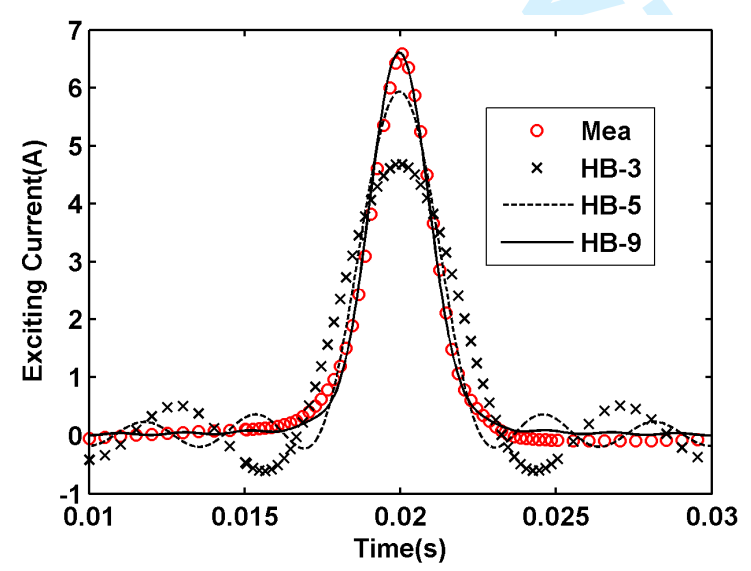

Figure 3. Exciting currents under different DC bias conditions, $\left(I_{d c}=0.847 \mathrm{~A} ; U_{a c}=320 \mathrm{~V}\right)$

Table II Calculation results under different DC bias excitations

\begin{tabular}{c|c|c|c|c|c|c|c|c}
\hline \hline$I_{\mathrm{dc}}(\mathrm{A})$ & $U_{\mathrm{ac}}(\mathrm{V})$ & $N$ & $P_{\mathrm{c}}$ & $T_{\mathrm{c}}(\mathrm{s})$ & $I_{\mathrm{rms}, \mathrm{c}}(\mathrm{A})$ & $I_{\mathrm{rms}, \mathrm{m}}(\mathrm{A})$ & $\varepsilon$ & Error $(\%)$ \\
\hline 0.4256 & 370 & 9 & 170 & 5678.5 & 1.2895 & 1.3486 & 0.015 & 4.38 \\
\hline 0.4256 & 370 & 7 & 158 & 3303.6 & 1.2813 & 1.3486 & 0.01 & 4.99 \\
\hline \hline
\end{tabular}




\begin{tabular}{c|c|c|c|c|c|c|c|c}
\hline \hline 0.847 & 240 & 5 & 171 & 1888.6 & 2.1202 & 2.3463 & 0.01 & 9.64 \\
\hline 0.847 & 240 & 9 & 174 & 5788.2 & 2.3516 & 2.3463 & 0.015 & -0.23 \\
\hline \hline
\end{tabular}

Table III Comparison of the computational cost between the two methods

\begin{tabular}{ccccccccccc}
\hline \hline & & & & \multicolumn{3}{c}{ Conventional HB } & \multicolumn{3}{c}{ Decomposed HB } \\
$I_{\mathrm{dc}}(\mathrm{A})$ & $U_{\mathrm{ac}}(\mathrm{V})$ & $\varepsilon$ & $N$ & & & & & & \\
& & & & $P_{\mathrm{c}, 1}$ & $T_{\mathrm{c}, 1}(\mathrm{~s})$ & $M_{1}(\mathrm{Mb})$ & $P_{\mathrm{c}, 2}$ & $T_{\mathrm{c}, 2}(\mathrm{~s})$ & $M_{2}(\mathrm{Mb})$ \\
0.4256 & 370 & 0.015 & 8 & 53 & 3062.1 & 10.98 & 164 & 4409.1 & 0.47 \\
0.847 & 320 & 0.015 & 9 & 72 & 4287.3 & 13.61 & 189 & 5943.5 & 0.47 \\
\hline \hline
\end{tabular}

From Table II it can be concluded that at least seven harmonics should be truncated in the computation to control the errors of the exciting current within 5\%. The number of iterations for convergence depends on the DC bias excitation, truncated harmonic number $N$ and stopping criterion. The increased $\left(I_{\mathrm{dc}}\right.$ or $\left.U_{\mathrm{ac}}\right)$ indicates a stronger nonlinearity of the magnetic core in computation, and more iterative steps will be required to obtain convergent harmonic solutions. A larger truncated harmonic number also results in more iterative steps. If the stopping criterion is set reasonably, small errors and less iterative steps can be achieved simultaneously.

The decomposed HBFEM is compared with the conventional approach (in terms of memory requirement and computational time) in Table III. The symbol $M$ represents the memory that the system matrix requires in the computation. $T_{\mathrm{c}}$ is the computational time. It is apparent that the conventional HBFEM requires a large memory in the calculation, which may limit its application in large-scale computation, such as 3-D problems. The decomposed method has significantly reduced the memory cost. An increase in computational time is due to the sequential solving scheme of each harmonic solution. In fact, each harmonic solution in equation (5) can be solved in parallel by using the block Jacobi algorithm. In that case, the three-dimensional DC-biasing problems in the power transformer can be solved more efficiently.

\section{Analysis of the flux density and flux distribution under DC bias conditions}

One point on the cross section of the LCM shown in Figure 4, such as point B, can be selected to observe the effect of DC bias on the total flux. The flux density $B_{x}$ in point $\mathrm{B}$ is calculated by the decomposed HBFEM and the time domain finite element method (TDFEM) respectively $\left(B_{y}\right.$ is too small to be analyzed). The waveforms of flux density and the corresponding exciting current shown in Figure 5(a) and 5(b) respectively demonstrate that the DC-biasing magnetic fields calculated by the two methods coincide well. The waveform of reluctivity under DC bias condition is presented in Figure 5(c). Due to the existence of DC bias in the exciting coil, the total flux is raised by the DC flux and saturates the magnetic core in one direction. This corresponds to the exciting current distorted in the half-cycle shown in Figure 3. Table III presents the calculated harmonic components of flux density $\left(B_{x}\right)$ and reluctivity $(v)$. 


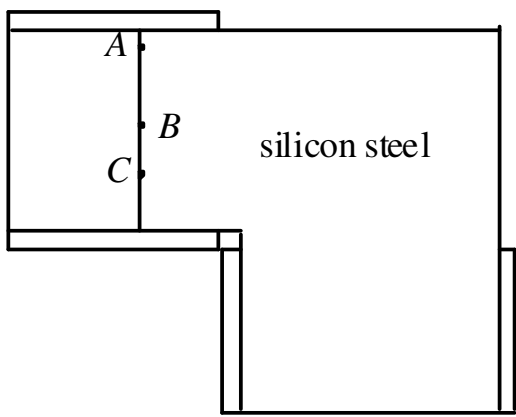

Figure 4. Computational region: one quarter of the square laminated core model

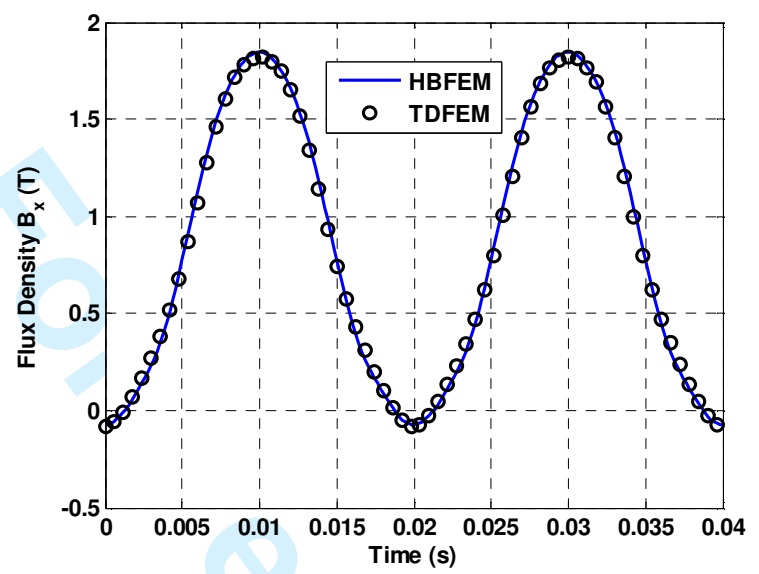

(a) Waveform of flux density $B_{x}$ in point B

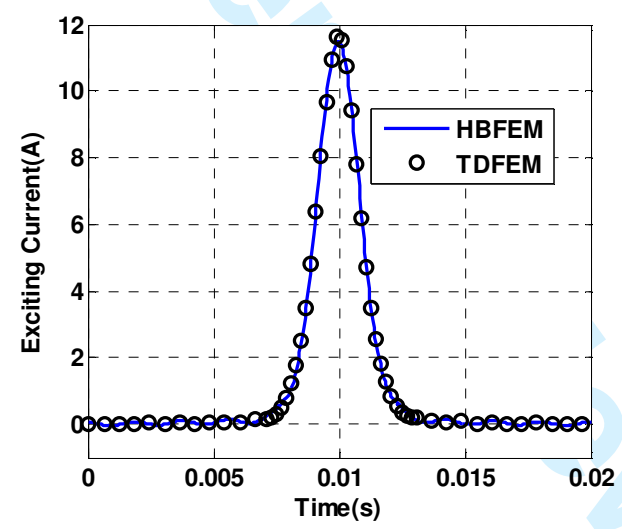

(b) Exciting current

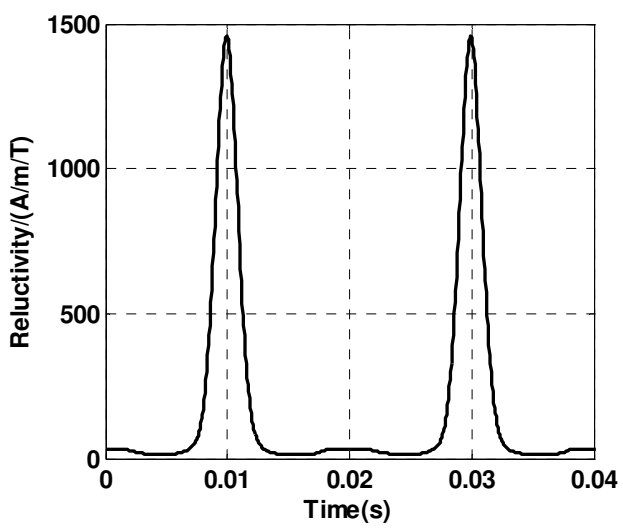

(c) Waveform of reluctivity 
Figure 5. Calculated flux density, exciting current and reluctivity $\left(I_{d c}=1.27 \mathrm{~A}, U_{a c}=370 \mathrm{~V}\right)$

Table IV Harmonic components of $B_{x}$ and reluctivity in point B

\begin{tabular}{|c|c|c|c|c|c|c|c|c|c|c|}
\hline $\begin{array}{l}\text { Harmonic } \\
\text { components }\end{array}$ & $B_{\mathrm{x} 0}$ & $B_{\mathrm{x} 1}$ & $B_{\mathrm{x} 2}$ & $B_{\mathrm{x} 3}$ & $B_{\mathrm{x} 4}$ & $B_{\mathrm{x} 5}$ & $B_{\mathrm{x} 6}$ & $B_{\mathrm{x} 7}$ & $B_{\mathrm{x} 8}$ & $B_{\mathrm{x} 9}$ \\
\hline Flux density $(\mathrm{T})$ & 0.9256 & 0.9847 & 0.0509 & 0.0299 & 0.0122 & 0.0016 & 0.0045 & 0.0015 & 0.0019 & 0.0013 \\
\hline $\begin{array}{l}\text { Harmonic } \\
\text { components }\end{array}$ & $v_{0}$ & $v_{1}$ & $v_{2}$ & $v_{3}$ & $v_{4}$ & $v_{5}$ & $v_{6}$ & $v_{7}$ & $v_{8}$ & $v_{9}$ \\
\hline $\begin{array}{l}\text { Reluctivity } \\
(\mathrm{A} /(\mathrm{m} \cdot \mathrm{T}))\end{array}$ & 184.50 & 5.11 & -308.54 & -8.80 & 280.77 & 11.36 & -218.99 & -12.01 & 165.67 & 10.93 \\
\hline $\begin{array}{l}\text { Harmonic } \\
\text { components }\end{array}$ & $v_{10}$ & $v_{11}$ & $v_{12}$ & $v_{13}$ & $v_{14}$ & $v_{15}$ & $N_{16}$ & $v_{17}$ & $v_{18}$ & $v_{19}$ \\
\hline $\begin{array}{l}\text { Reluctivity } \\
(\mathrm{A} /(\mathrm{m} \cdot \mathrm{T}))\end{array}$ & -115.35 & -9.49 & 73.55 & 7.28 & -45.71 & -5.10 & 24.57 & 3.64 & -12.79 & -2.18 \\
\hline
\end{tabular}

Note: $B_{x 0}=\left|B_{x, 0}\right|$ and $B_{x i}=\sqrt{B_{x, 2 i-1}^{2}+B_{x, 2 i}^{2}}(i=1,2, \ldots, 9)$.

The DC flux is not only affected nonlinearly by the DC bias, but it also depends on the alternating voltage. Meanwhile, the AC flux varies with different DC bias conditions. Harmonic components of $B_{x}$ in point $\mathrm{B}$ are observed under different DC bias conditions. As Table III shows, the DC and fundamental components are dominant in flux density $B_{x}$. Therefore, four different DC bias levels and five different $\mathrm{AC}$ excitation cases (shown in Table $\mathrm{V}$ and Table VI) are integrated to analyze the variation of the DC component $B_{x 0}$ and the fundamental component $B_{x 1}$ under DC bias excitation.

Table V DC flux density $\left(B_{\mathrm{x} 0}\right)$ under different DC bias and AC excitations

\begin{tabular}{ccccccc}
\hline \hline \multirow{2}{*}{$B_{\mathrm{dc}}(\mathrm{T})$} & \multicolumn{5}{c}{$U_{\mathrm{ac}}(\mathrm{V})$} \\
\cline { 2 - 7 } & & 26 & 133 & 238 & 370 & 420 \\
\hline \multirow{4}{*}{$I_{\mathrm{dc}}(\mathrm{A})$} & 0.426 & 1.6489 & 1.4256 & 1.1442 & 0.8410 & 0.7201 \\
& 0.847 & 1.7176 & 1.4955 & 1.2037 & 0.8981 & 0.7846 \\
& 1.274 & 1.7529 & 1.5324 & 1.2647 & 0.9256 & 0.8126 \\
& 1.691 & 1.7757 & 1.5529 & 1.3067 & 0.9308 & 0.8298 \\
\hline
\end{tabular}

Table VI AC flux density $\left(B_{x 1}\right)$ under different DC bias and AC excitations

\begin{tabular}{ccccccc}
\hline \hline \multirow{2}{*}{$B_{\text {ac }}(\mathrm{T})$} & \multicolumn{5}{c}{$U_{\text {ac }}(\mathrm{V})$} \\
\cline { 3 - 7 } & & 26 & 133 & 238 & 370 & 420 \\
\hline \multirow{4}{*}{$I_{\mathrm{dc}}(\mathrm{A})$} & 0.426 & 0.0688 & 0.3819 & 0.6948 & 0.9774 & 1.0967 \\
& 0.847 & 0.0699 & 0.3768 & 0.6994 & 0.9835 & 1.0832 \\
& 1.274 & 0.0696 & 0.3778 & 0.6952 & 0.9846 & 1.0951 \\
& 1.691 & 0.0703 & 0.3779 & 0.6892 & 0.9902 & 1.0768 \\
\hline \hline
\end{tabular}




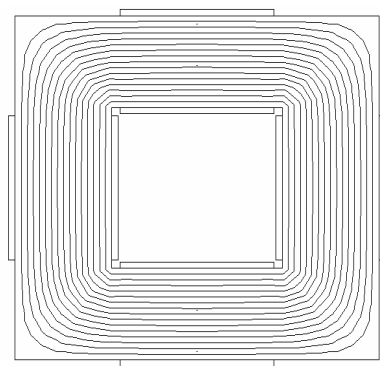

a. DC flux

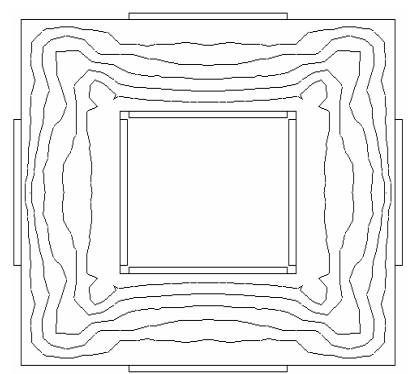

d. Third order $(3 \omega t=\pi / 2)$

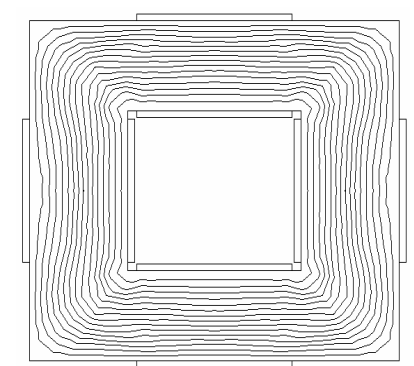

b. Fundamental $(\omega t=\pi / 2)$

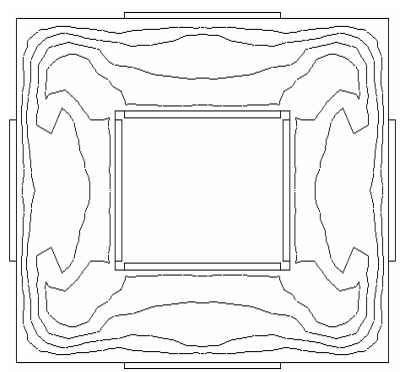

e. Fourth order $(4 \omega t=\pi / 2)$

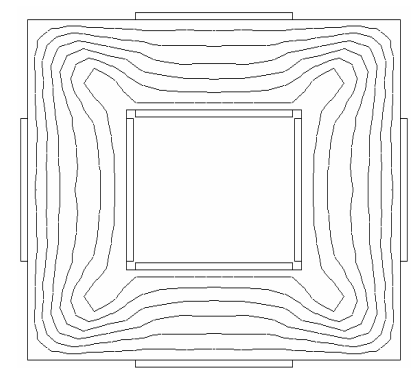

c. Second order $(2 \omega t=\pi / 2)$

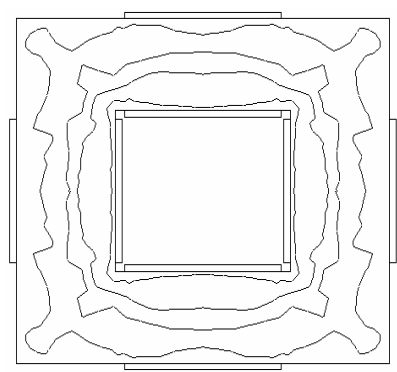

f. Fifth order $(5 \omega t=\pi / 2)$

Figure 6. Harmonic flux distributions $\left(I_{d c}=1.27 \mathrm{~A}, U_{a c}=370 \mathrm{~V}\right)$

Table $\mathrm{V}$ shows that when the alternating voltage $U_{\text {ac }}$ is kept constant, the DC flux density increases slowly and tends to be constant with the increment of DC bias $I_{\mathrm{dc}}$. When the DC bias is kept constant, the AC excitation will suppress the DC flux density nonlinearly. It can also be concluded from Table VI that the DC bias has almost no influence on the AC flux density, whereas the AC excitation gives rise to the variation of AC flux density.

Harmonic flux distributions under DC bias conditions are presented in Figure 6. The harmonic flux distributions vary with the time (related to phase angle) and excitations (related to DC bias and alternating voltage). The high-order harmonic distribution is apparently different from the fundamental and DC component under DC bias conditions. That may affect the total flux, which is the superposition of all harmonic components.

\section{Conclusion}

The decomposed HBFEM combined with the Gauss-Seidel iterative scheme is presented to investigate the DC bias phenomena in saturated electromagnetic devices. The DC flux raises the total flux and saturates the ferromagnetic core rapidly and significantly in one direction. The DC flux is affected by DC bias and alternating voltage nonlinearly and simultaneously, while AC flux varies with alternating voltage. Harmonic flux distributions under DC bias conditions reflect the incidence of saturation of the ferromagnetic core. The harmonic solutions are solved separately and sequentially by the decomposed HBFEM. Compared with the conventional approach, the decomposed method can significantly decrease the memory requirement and is applicable to large-scale computation of DC-biasing magnetic fields. If the block Jacobi iterative scheme is employed to solve the equation (5), each harmonic solution can be calculated in parallel. Therefore, the decomposed HBFEM can be used in 3-D problems of power transformers. 


\section{References}

Ausserhofer S., Biro, O. and Preis, K. (2007), "An efficient harmonic balance method for nonlinear eddy current problems”, IEEE Trans. on Mag., Vol. 43 No. 4, pp. 1229-32.

Bachinger, F., Langer, U. and Schoberl, J. (2006), "Efficient solvers for nonlinear time-periodic eddy current problems", Comput. Visual. Sci., Vol 9 No 4, pp: 197-207.

Biro, O., Buchgraber, G., Leber, G. and Preis, K. (2008), "Prediction of magnetizing current waver-formers in a three-phase power transformer under DC bias", IEEE Trans. on Mag., Vol. 44 No. 6, pp. 1554-7.

Biro, O. and Preis, K. (2006), "An efficient time domain method for nonlinear periodic eddy current problems", IEEE Trans. on Mag., Vol. 42 No. 4, pp. 695-8.

Cheng, Z., Norio, T. and Behzad, F. (2009), Electromagnetic and Thermal Field Modeling and Application in Electrical Engineering, Science Press, Beijing, China.

Ciric I. R. and Hantila, F. I. (2007), "An efficient harmonic method for solving nonlinear time-periodic eddy-current problems", IEEE Trans. on Mag., Vol. 43 No. 4, pp. 1185-8.

Deblecker, O. and Lobry, J. (2006), "A new efficient technique for harmonic-balance finite-element analysis of saturated electromagnetic devices", IEEE Trans. on Mag., Vol. 42 No. 4, pp. 535-8.

Gyselinck, J., Dular, P., Geuzaine, C. and Legros, W. (2002), "Harmonic-balance finite-element modeling of electromagnetic devices: A novel approach”, IEEE Trans. on Mag., Vol. 38 No. 2, pp. 521-4.

Lombard, P. and Meunier, G. (1992), "General method for electric and magnetic coupled problem in 2D and magnetodynamic domain”, IEEE Trans. on Mag., Vol. 28 No. 2, pp. 1291-4.

Lu, J., Yamada, S. and Harrison, H. B. (1996), "Application of harmonic balance-finite element method (HBFEM) in the design of switching power supplies", IEEE Trans. on Power Electronics, Vol. 11 No. 2, pp. 347-55.

Picher, P., Bolduc, L., Dutil, A. and Pham, V. Q. (1997), "Study of the acceptable DC current limit in core-form power transformers", IEEE Trans. on Power Delivery, Vol. 12 No. 1, pp. 257-65.

Pirjola, R. (2000), "Geomagnetically induced current during magnetic storms", IEEE Trans. Power Apparatus and Systems, Vol. 104 No. 1, pp. 212-219.

Rizzoli, V., Masotti, D. and Mastri, F. (1995), "General-purpose analysis of nonlinear circuits containing saturating/hysteresis inductor by the harmonic-balance technique", IEEE Trans. on Mag., Vol. 31 No. 3, pp. 2290-2302.

Saad, Y. (2009), Iterative methods for sparse linear systems, Science Press, Beijing, China.

Yamada, S., Biringer, P. and Bessho, K. (1991), "Calculation of nonlinear eddy-current problems by the harmonic balance finite element method", IEEE Trans. on Mag., Vol. 27 No. 5, pp. 4122-5.

Yamada, S. and Bessho, K. (1988), "Harmonic field calculation by the combination of finite element analysis and harmonic balance method", IEEE Trans. on Mag., Vol. 24 No. 6, pp. 2588-90.

Yamada, S., Bessho, K. and Lu, J. (1989), "Harmonic balance finite element method applied to nonlinear AC magnetic analysis”, IEEE Trans. on Mag., Vol. 25 No. 4, pp. 2971-3.

Yamada, S., Biringer, P., Hirano, K. and Bessho, K. (1990), "Finite element analysis of nonlinear 
dynamic magnetic field with DC component in the harmonic domain", IEEE Trans. on Mag., Vol. 26 No. 5, pp. 2199-201.

Yao, Y., Koh, C. S., Ni, G. and Xie, D. (2005), "3-D nonlinear transient eddy current calculation of online power transformer under DC bias”, IEEE Trans. on Mag., Vol. 41 No. 5, pp. 1840-3.

Zhang, B., Cui, X., Zeng, R. and He, J. (2006), "Calculation of DC current distribution in AC power system near HVDC system by using moment method coupled to circuit equations", IEEE Trans. on Mag., Vol. 42 No. 4, pp. 703-6.

Zhao, X., Lu, J., Li, L., Cheng, Z. and Lu, T. (2010), "Analysis of the DC bias phenomenon by the harmonic balance finite element method", IEEE Trans. on Power Delivery, accepted. 
Response to referee 1:

Thanks for your most valuable comments on improvement of the submitted paper. The relevant revisions has been completed in the revised paper and listed below:

1. The mathematics in section 2 and 3 has been simplified as much as possible.

2. In this paper the DC component is solved together with fundamental component. The DC component can also be solved separately. In that case, the relucitivity matrices should be changed, but the final solution will not be affected.

3. The magnetic core is laminated by silicon steel sheets, and there are minor gaps between them. In order to obtain the accurate magnetizing curve of LCM, some measurements have been done. Please see the section 5.1 of the revised paper. Detailed description of the experiments was given in section 5.2, together with the added figure in Figure 1, which is a supplement of Figure 2 (the electric diagram). Figure 3 has been revised, and different harmonics are truncated in computation to observe the effect of $N$ (truncated harmonic number) on the calculated exciting current.

4. The electric circuit that exciting coil belongs to is simulated. The DC voltage is obtained from DC bias current $I_{\mathrm{dc}}$ and the resistance $R$ of exciting coil, which is explained at the end of section 5.2 of the revised paper.

5. The calculated exciting currents that corresponds to magnetic induction in Figure 5 has been presented, please see the Figure 5(b) of the revised paper.

6. Figure 6 and Figure 7 in the original paper have been deleted, and the calculated data are listed in Table $\mathrm{V}$ and Table VI of the revised paper.

7. The word "disorder" (concerning Figure 8) is not explicit, and the explanation is revised in the original sentence (in the last paragraph in section 6). High-order harmonic flux distribution is different with the DC and fundamental components, which may affect the total flux. In fact the waveform of flux density in Figure 5(a) has been distorted slightly.

8. A new stopping criterion has been presented in section 4 in the revised paper and the corresponding total number of iterations has been reduced. The conventional HB method is compared with the decomposed method in detail in Table III.

9. The language errors in the paper have been corrected by a native English speaker.

Response to referee 2 :

1. The main contribution of the submitted paper is to reduce the memory cost of HB method in computation and improve the solution efficiency by the Gauss-Seidel algorithm. The originality of the submitted paper has been emphasized in the introduction according to your suggestion. In addition the two papers have been compared by editors and the originality is checked. Please see relevant revision in the Paragraph 1 and 2 in section 1.

2. The paper "General-purpose analysis of nonlinear circuits containing saturating/hysteretic inductors by the harmonic-balance technique" has been added in the reference list, thanks a lot for your suggestion.

3. The size of the FE problem has been given in the Paragraph 1, section 5.3. Table II presents the number of iterative steps the corresponding execution time within the prescribed tolerance. 
4. The saturation curve of the magnetic core has been given in section 5.1 and Figure 1(c).

5. The conventional HB method is compared with the decomposed HB method, please see the Table III.

6. Equation (1) are revised and given directly for the 2D case with a distinction between forced and eddy-current density. The weak form of equation (1) based on Galerkin's method has been given in equation (4).

Response to referee 3:

1. The relaxation factor $\alpha$ is related to the convergence of harmonic solutions. Zhiguang Cheng and Takahashi Norio suggest $\alpha$ set to be 0.2 or 0.3 in their newly published book "Electromagnetic and thermal field modeling and application in electrical engineering", which is also listed in the reference list of the paper. In fact a larger or smaller value of alpha will result in the deficiency of the convergence and even non-convergence. Therefore in the paper the authors suggest alpha set to be 0.2 or 0.3 , please see the Paragraph 1, section 5.3.

2. The stopping criterion has been changed in the revised paper, as follows

$$
\frac{\left\|\boldsymbol{X}^{p+1}-\boldsymbol{X}^{p}\right\|}{\left\|\boldsymbol{X}^{p}\right\|}<\varepsilon,
$$

where $\boldsymbol{X}$ include all harmonic solutions of magnetic vector potential $\boldsymbol{A}$ and current density $\boldsymbol{J}$. The new criterion deletes the redundant iterative steps in computation, compared with the original one. Please see the end of section 4.2 and Table II in the revised paper. The initial value of magnetic vector potential $\boldsymbol{A}$ can be assumed to be $\mathbf{0}$.

3. In the provided example (DC-biasing problem), the authors have chosen 9 harmonics in computation to control the error of calculated exciting current within 5\%. Different results have been given in Table II and waveforms of exciting current are presented in Figure 3 to observe the effect of $N$ on the computational exciting current. The flux density mainly contains DC component and fundamental component, however, inaccuracy of exciting current may affect the calculated flux density, since they are related through the B-H curve.

4. The conventional HB method is compared with the decomposed HB method, please see the Table III. The size of the FE problem is given in Paragraph 1, section 5.3.

5. The revised paper has been proofread by a native English speaker.

Response to referee 4:

1. The advantage of HBFEM includes powerful harmonic analysis, resolution of widely separated harmonic problems, analysis of resonant frequency of mechanical vibration in the DC biased transformer, computation of frequency power loss, and easy implementation of parallel algorithm, etc. Authors will make use of these advantages to investigate the DC biased problem in saturated electromagnetic devices at next step. In this paper we mainly focus on comparison between conventional $\mathrm{HB}$ method and the proposed decomposed method and analysis of DC bias phenomenon on the LCM. A detailed comparison between HBFEM and other methods can be given as follows,

\begin{tabular}{|l|l|l|l|}
\hline Frequency-domain & Step-by-step & Time-periodic & Harmonic balance \\
\hline
\end{tabular}




\begin{tabular}{|c|c|c|c|c|}
\hline & method & mehtod & method & method \\
\hline $\begin{array}{l}\text { Computation } \\
\text { domain }\end{array}$ & $\begin{array}{l}\text { Single frequency } \\
\text { domain }\end{array}$ & Time domain & Time domain & $\begin{array}{l}\text { Multiple frequency } \\
\text { domain }\end{array}$ \\
\hline $\begin{array}{l}\text { For nonlinear } \\
\text { and harmonic } \\
\text { problems }\end{array}$ & $\begin{array}{l}\text { Yes, for weak } \\
\text { nonlinear fields but } \\
\text { not for harmonic } \\
\text { problems }\end{array}$ & $\begin{array}{l}\text { Yes, for weak } \\
\text { nonlinear fields } \\
\text { and harmonic } \\
\text { problems }\end{array}$ & $\begin{array}{l}\text { Yes, for weak } \\
\text { nonlinear fields } \\
\text { and harmonic } \\
\text { problems }\end{array}$ & $\begin{array}{l}\text { Yes, for weak and } \\
\text { strong nonlinear } \\
\text { fields } \\
\text { harmonic } \\
\text { problems }\end{array}$ \\
\hline $\begin{array}{l}\text { Widely separated } \\
\text { harmonics }\end{array}$ & Cannot compute & $\begin{array}{l}\text { Difficult to } \\
\text { compute }\end{array}$ & $\begin{array}{l}\text { Difficult to } \\
\text { compute }\end{array}$ & Easy to compute \\
\hline $\begin{array}{l}\text { Computation } \\
\text { time depending } \\
\text { on }\end{array}$ & $\begin{array}{l}\text { Number of degrees of } \\
\text { freedom }\end{array}$ & $\begin{array}{ll}\text { Time step } & \text { and } \\
\text { number } & \text { of } \\
\text { degrees } & \text { of } \\
\text { freedom } & \end{array}$ & $\begin{array}{ll}\text { Time step } & \text { and } \\
\text { number } & \text { of } \\
\text { degrees } & \text { of } \\
\text { freedom } & \end{array}$ & $\begin{array}{l}\text { Harmonic number } \\
\text { and number of } \\
\text { degrees of freedom }\end{array}$ \\
\hline $\begin{array}{l}\text { Computation } \\
\text { accuracy }\end{array}$ & $\begin{array}{l}\text { Large error rate at } \\
\text { high frequency } \\
\text { harmonics }\end{array}$ & $\begin{array}{l}\text { Truncation } \\
\text { error }\end{array}$ & Truncation error & $\begin{array}{l}\text { Number } \\
\text { harmonics } \\
\text { considered }\end{array}$ \\
\hline $\begin{array}{l}\text { Calculation of } \\
\text { harmonic } \\
\text { components }\end{array}$ & Impossible & $\begin{array}{l}\text { Calculate from } \\
\text { computation } \\
\text { results }\end{array}$ & $\begin{array}{l}\text { Calculate from } \\
\text { computation } \\
\text { results }\end{array}$ & $\begin{array}{l}\text { Calculate several } \\
\text { harmonics } \\
\text { simultaneously } \\
\text { (the result itself) }\end{array}$ \\
\hline $\begin{array}{l}\text { Post-processing } \\
\text { of harmonics }\end{array}$ & Impossible & Indirectly & Indirectly & directly \\
\hline $\begin{array}{l}\text { Applicable in } \\
\text { concurrent } \\
\text { computation }\end{array}$ & Not easy & Not easy & & Easy \\
\hline
\end{tabular}

2. The 3-D program of HBFEM is in development, and it will be used to analyze the three phase power transformer in the future work.

Response to referee 5:

1. Three papers relevant to "fixed-point technique and harmonic balance method" have been acknowledged in the paper, thanks for your valuable suggestion.

2. Equations (2)-(5) have been simplified and other equations are also simplified as much as possible. The HB method can be expressed by real notation or complex notation. The real notation is retained in this paper, because if the complex notation is used in the paper, all other expressions such as reluctivity matrix and harmonic matrix should be changed. What's more, if the complex number is introduced, the computational program is also different. In our computational program the algorithm in accordance with real notation is used.

3. The exposition is improved so that the method is applicable to any type elements in two-dimensional case. Please see equations (7)-(10) in the revised paper. The weak form of 
equation (1) has been given in equation (4). The equation set shown in (5) can be obtained by substituting all harmonic variables in (2) and (3) into equation (4). There is an error in the second sum sign in (7) in the original paper, and it has been corrected in the revised paper. Please see the equation (5) and the expressions below equation (16) in the revised paper. Thanks for your comments.

a) Exposition related to $\boldsymbol{A}$ and $\boldsymbol{K}$ has been revised to be applicable to any type of elements. Please see equation (6) and (7) in the revised paper. Thanks for your comments.

b) Expressions such as "self-correlation reluctivity matrix", "cross-correlation reluctivity matrix", "coefficient matrix relevant to eddy current" and "coefficient matrix relevant to magnetic reluctivity" have been avoided.

c) The "reluctivity matrix" in equation (11)-(14) in the revised paper are derived from the multiplication of magnetic vector potential $A$ and reluctivity $v$. The paper "Calculation of nonlinear eddy-current problems by the harmonic balance finite element method" published by Yamada S. and Biringer P.P. in 1991 can be regarded as a reference. It is mentioned in the last Paragraph, Page 3 in the revised paper.

4. Why complex notations are not used by authors in this paper has been explained in part 2 above. The representation of the equations by real notation has been simplified in the revised paper. Thank you for your suggestion and the computations in complex notations and in real notations will be performed respectively and compared in the subsequent work.

5. The original explanation is not exact and has been corrected in the section 4.2 in the revised paper. The harmonic components of reluctivity have been presented in Table IV.

6. a) The authors appreciate your instructive suggestions. The eddy current in the thin steel strip can be neglected in computation. In this paper the laminated core model is used to verify the effectiveness and validity of the proposed method. In addition the authors now do not have a new model with eddy current to carry out the relevant experiment. The 3-D program is in development, and the eddy current problem in DC bias phenomenon can be future discussed in three phase power transformers.

b) "The number of iterations for convergence depends on the DC bias excitation, truncated harmonic number $N$ and stopping criterion". This sentence has been explained in detail in Paragraph 3, section 5.3, together with Table II in the revised paper.

c) The stopping criterion has been revised in section 4.2. The original criterion is to observe numerical error on each node, while the new one is to observe the error of the norm of all harmonic solutions. The newly presented methods delete a large number of redundant iterative steps. Please see Table II and Table III. Actually the decomposed equation set in (5) can also be solved parallel by block Jacobi algorithm. A cluster can be used to solve the 3-D problems by using the concurrent computation. Please see Paragraph 4, section 5.3.

d) The accepted paper by IEEE is provided to editors and compared with the submitted paper to COMPEL, the originality has been checked.

7. The grammar and spelling check has been done by a native English speaker.

8. The authors appreciate your reminding of application of fixed-point technique in the FEM. Biro, Ausserhofer, Hantila, Ciric and other outstanding researchers have done a lot of work in this field. We are also highly interested in that and have developed the fix-point HBFEM, in which a global and local convergent algorithm can be used. A new paper will be submitted to the COMPUMAG 2011. The authors would like to have a further discussion with you in aspects 
of the application of fixed-point technique and other numerical technique.

1

6

7

8

9

10

11

12

13

14

15

16

17

18

19

20

21

22

23

24

25

26

27

28

29

30

31

32

33

34

35

36

37

38

39

40

41

42

43

44

45

46

47

48

49

50

51

52

53

54

55

56

57

58

59

60 